\title{
Immunogenicity of CAR T cells in cancer therapy
}

\author{
Dimitrios L. Wagner(10 1,2,17, Enrico Fritsche ${ }^{1,17}$, Michael A. Pulsipher ${ }^{3}$, Nabil Ahmed $^{4,5}$, \\ Mohamad Hamieh 6 , Meenakshi Hegde ${ }^{4,5}$, Marco Ruella ${ }^{7,8}$, Barbara Savoldo ${ }^{9}$, \\ Nirali N. Shah ${ }^{10}{ }^{10}$, Cameron J. Turtle ${ }^{11,12}$, Alan S. Wayne ${ }^{13,14}$ and \\ Mohamed Abou-el-Enein 1,15,16凶
}

\begin{abstract}
Patient-derived T cells genetically reprogrammed to express CD19-specific chimeric antigen receptors (CARs) have shown remarkable clinical responses and are commercially available for the treatment of patients with certain advanced-stage B cell malignancies. Nonetheless, several trials have revealed pre-existing and/or treatment-induced immune responses to the mouse-derived single-chain variable fragments included in these constructs. These responses might have contributed to both treatment failure and the limited success of redosing strategies observed in some patients. Data from early phase clinical trials suggest that CAR T cells are also associated with immunogenicity-related events in patients with solid tumours. Generally, the clinical implications of anti-CAR immune responses are poorly understood and highly variable between different CAR constructs and malignancies. These observations highlight an urgent need to uncover the mechanisms of immunogenicity in patients receiving CAR T cells and develop validated assays to enable clinical detection. In this Review, we describe the current clinical evidence of anti-CAR immune responses and discuss how new CAR T cell technologies might impact the risk of immunogenicity. We then suggest ways to reduce the risks of anti-CAR immune responses to CAR T cell products that are advancing towards the clinic. Finally, we summarize measures that investigators could consider in order to systematically monitor and better comprehend the possible effects of immunogenicity during trials involving CAR T cells as well as in routine clinical practice.
\end{abstract}

Chimeric antigen receptors (CAR) are fusion proteins that redirect $\mathrm{T}$ cell specificity towards surface molecules expressed on tumour cells independently of the conventional T cell receptor (TCR)-major histocompatibility complex (MHC) interactions. CARs are introduced into $\mathrm{T}$ cells through gene transfer ${ }^{1,2}$. The antigen-recognition domain most often consists of a mouse-derived monoclonal antibody as a continuous peptide single-chain variable fragment $(\mathrm{scFv})$ steered through an extracellular spacer domain that provides flexibility. ScFvs engage with their target epitopes and confer activation signals through modular intracellular signalling domains. Currently, CAR T cells are generated ex vivo from peripheral blood-derived $\mathrm{T}$ cells, which are typically transduced with replication-deficient vectors that integrate the CAR expression cassette into the T cell genome. These CAR T cells are subsequently expanded to large numbers in culture. After infusion into patients, these cells can recognize and eliminate tumour cells expressing the target antigen.
Autologous (patient-derived) and, occasionally, allogeneic (donor-derived) CAR T cells have successfully progressed from preclinical to clinical development and are currently used to treat patients with cancer ${ }^{3-5}$. The most successful CAR T cell products to date, of which three have received FDA approval (tisagenlecleucel, axicabtagene ciloleucel and brexucabtagene autoleucel), target the B cell lineage antigen CD19. CD19-targeted CAR T cells have become an important treatment option for patients with acute B cell lymphoblastic leukaemia (B-ALL) or certain aggressive B cell non-Hodgkin lymphomas (NHLs), inducing complete remissions in a subset of heavily pre-treated patients with extensive disease. These CAR T cells are also associated with cytokine-release syndrome, immune effector cell-associated neurotoxicity syndrome and other immune-mediated adverse events ${ }^{6}$. To date, the success of CAR T cells in patients with haematological malignancies has not been replicated in patients with solid tumours owing to several barriers, which have abouelenein@med.usc.edu https://doi.org/10.1038/ s41571-021-00476-2 


\section{Key points}

- Pre-existing and/or treatment-induced immunity to chimeric antigen receptor (CAR) constructs containing mouse-derived single-chain variable fragments are associated with treatment failure in certain patients and might limit the success of redosing strategies.

- The possible effects of immunogenicity on CAR T cell persistence and function are currently poorly understood.

- Novel technologies designed to enhance CAR T cell performance and/or the application of allogeneic CAR T cells might further amplify the likelihood of anti-CAR immune responses, thus necessitating strategies to overcome such risks.

- Various monitoring, mitigation and management approaches can be used to reduce the risk of anti-CAR immunity, although validated assays enabling adequate assessments of anti-CAR immune responses remain an unmet need.

- We advocate for the inclusion of CAR-associated immunogenicity analysis in both preclinical and clinical investigations of CAR T cell therapy.

been reviewed in detail elsewhere ${ }^{7,8}$. Therefore, current efforts aim to increase the potency of CAR T cells and/or to combine them with other treatments designed to overcome these barriers.

CAR T cells have the potential to induce both humoral and cellular anti-CAR immune responses to non-self components of the CAR construct or to residual proteins originating from the gene-transfer vectors, which are inherently immunogenic (FIG. 1). This reaction might, in turn, contribute to limiting the efficacy and thus inhibiting the success of subsequently administered CAR T cells ${ }^{9-11}$. Furthermore, the scFvs of many CARs currently in clinical development are derived from mouse or other non-human monoclonal antibodies

\section{Author addresses}

${ }^{1}$ Berlin Center for Advanced Therapies (BeCAT) and Berlin Institute of Health (BIH) Center for Regenerative Therapies (BCRT), Charité - Universitätsmedizin Berlin, Berlin, Germany. ${ }^{2}$ Institute of Transfusion Medicine, Charité - Universitätsmedizin Berlin, Berlin, Germany. ${ }^{3}$ Section of Transplantation and Cellular Therapy, Children's Hospital Los Angeles Cancer and Blood Disease Institute, USC Keck School of Medicine, Los Angeles, CA, USA.

${ }^{4}$ Texas Children's Cancer and Hematology Centers, Texas Children's Hospital, Houston, TX, USA.

${ }^{5}$ Center for Cell and Gene Therapy, Baylor College of Medicine, Houston, TX, USA. ${ }^{6}$ Center for Cell Engineering and Immunology Program, Sloan Kettering Institute, New York, NY, USA.

${ }^{7}$ Center for Cellular Immunotherapies, University of Pennsylvania Philadelphia, Philadelphia, PA, USA.

${ }^{8}$ Division of Hematology and Oncology, Hospital of the University of Pennsylvania, Philadelphia, PA, USA.

'Lineberger Comprehensive Cancer Center, University of North Carolina, Chapel Hill, NC, USA.

${ }^{10}$ Pediatric Oncology Branch, Center for Cancer Research, National Cancer Institute, National Institutes of Health, Bethesda, MD, USA.

${ }^{11}$ Clinical Research Division, Fred Hutchinson Cancer Research Center, University of Washington, Seattle, WA, USA.

${ }^{12}$ Department of Medicine, University of Washington, Seattle, WA, USA.

${ }^{13}$ Cancer and Blood Disease Institute, Division of Hematology-Oncology, Children's Hospital Los Angeles, Los Angeles, CA, USA.

${ }^{14}$ Norris Comprehensive Cancer Center, Keck School of Medicine, University of Southern California, Los Angeles, CA, USA.

${ }^{15}$ Division of Medical Oncology, Department of Medicine, and Department of Stem Cell Biology and Regenerative Medicine, Keck School of Medicine, University of Southern California, Los Angeles, CA, USA.

${ }^{16}$ Joint USC/CHLA Cell Therapy Program, University of Southern California, and Children's Hospital Los Angeles, Los Angeles, CA, USA.

${ }^{17}$ These authors contributed equally: Dimitrios L. Wagner, Enrico Fritsche.
(Supplementary Table 1). Pre-existing antibodies that broadly recognize the scFvs of mouse immunoglobulins, called human anti-mouse antibodies (HAMAs), have been detected in subsets of patients ${ }^{12,13}$. Human or humanized scFvs can also contain non-self sequences because the variable binding fragments are generated through multiple gene recombination events and somatic hypermutation. Antibodies directed towards a specific antibody sequence (such as those contained in a human scFv) are known as anti-idiotype antibodies ${ }^{14,15}$. Moreover, the expression of proteins encoded by several human genes in a single peptide CAR chain creates fusion sequences at junctions that do not normally exist in humans. Thus far, no conclusive evidence exists that such anti-CAR immune responses contribute to other reported adverse events such as cytokine-release syndrome and immune effector cell-associated neurotoxicity syndrome; therefore, such toxicities are not the focus of this Review.

Cellular immune responses to genetically modified cytotoxic $\mathrm{T}$ cells have been documented in patients with cancer and in those with HIV, sometimes in association with immune rejection of the adoptively transferred T cells ${ }^{9-11}$. Particularly, T cells with specificity for the CAR transgene or residual proteins originating from gene-transfer vectors have been shown to deplete and inactivate the infused CAR T cells ${ }^{10}$. Similarly, the presence of humoral immunity and the development of antibodies to the CAR construct might interfere with CAR T cell activity by neutralizing the antigen-binding fragment and/or promoting early CAR T cell apoptosis ${ }^{16}$. A small risk of severe systemic anaphylaxis also exists owing to IgE-mediated HAMA-triggered mast cell degranulation after CAR $\mathrm{T}$ cell application in patients who are pre-sensitized to CAR T cells with mouse-derived scFvs and/or mouse-derived monoclonal antibodies ${ }^{17,18}$ (FIG. 1). Nevertheless, the effects of CAR-specific immunogenicity on the clinical outcomes after CAR $\mathrm{T}$ cell infusion remain both poorly understood and poorly investigated. Therefore, as new CAR constructs are developed to improve efficacy and persistence, understanding the origins and mechanisms of CAR T cell immunogenicity remains crucial, particularly in patients with solid tumours. Herein, we review published clinical data on immune responses to CAR $\mathrm{T}$ cells in patients with haematological or solid malignancies and discuss the strategies to investigate, mitigate and manage the risk of immunogenicity at different stages of CAR T cell product development.

\section{Clinical evidence of anti-CAR immunity} Antibodies to CD19-directed CARs do not appear to impair initial clinical responses. Pre-existing humoral immunity to CD19-specific CAR T cells has been observed with both tisagenlecleucel and axicabtagene ciloleucel ${ }^{19-22}$. The vast majority of patients with B-ALL $(84.6 \% ; n=88)^{22}$ who received tisagenlecleucel had preexisting anti-CAR antibodies, with a similarly high percentage $(91.4 \%)$ reported in patients with refractory diffuse large B cell lymphoma ${ }^{19}$. Treatment-emergent immunogenicity, defined as an increase in anti-CAR antibodies following infusion, has been reported in 
$5 \%$ and $36.7 \%$ of patients with diffuse large B cell lymphoma and B-ALL, respectively ${ }^{19,21}$. Far fewer patients receiving axicabtagene ciloleucel have been reported to have pre-existing anti-CAR antibodies $(3 \% ; n=94)^{20}$, while no pre-existing anti-CAR antibodies have been confirmed in patients receiving brexucabtagene autoleucel using a combination of two different assays ${ }^{23}$. All three of these products contain the same mouse-derived $\mathrm{scFv}$ (FMC63); therefore, the differences in the reported percentages of patients with pre-existing antibodies a

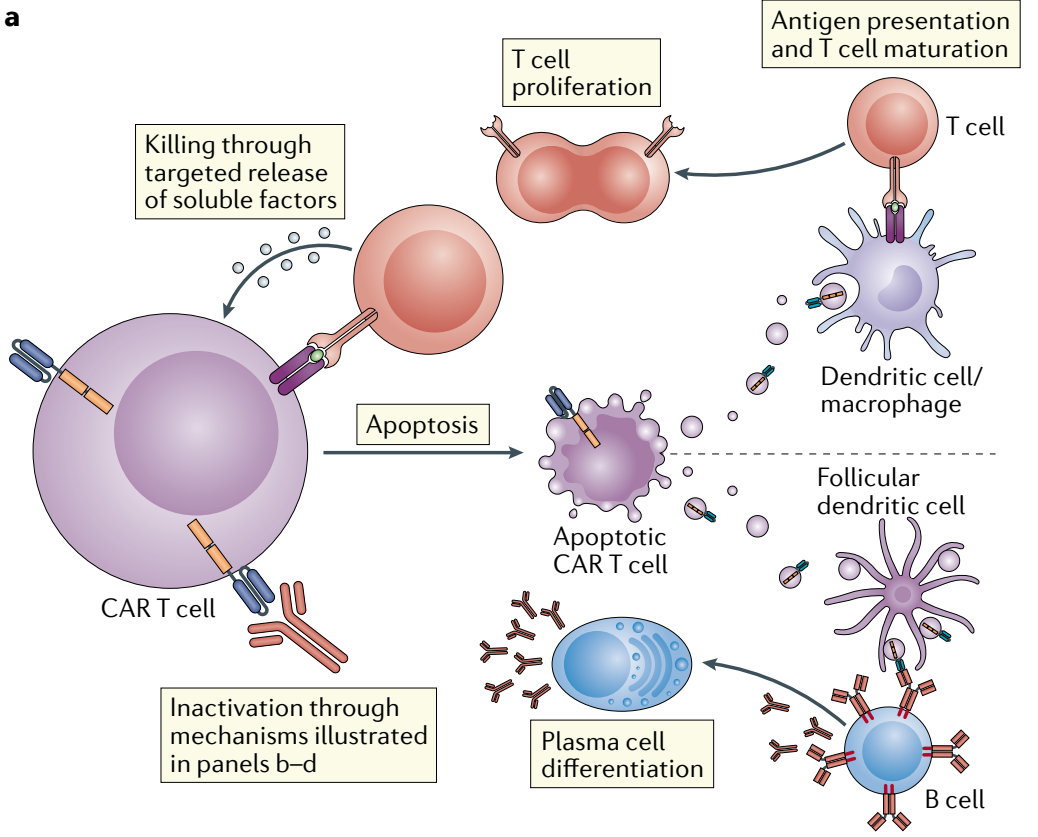

b

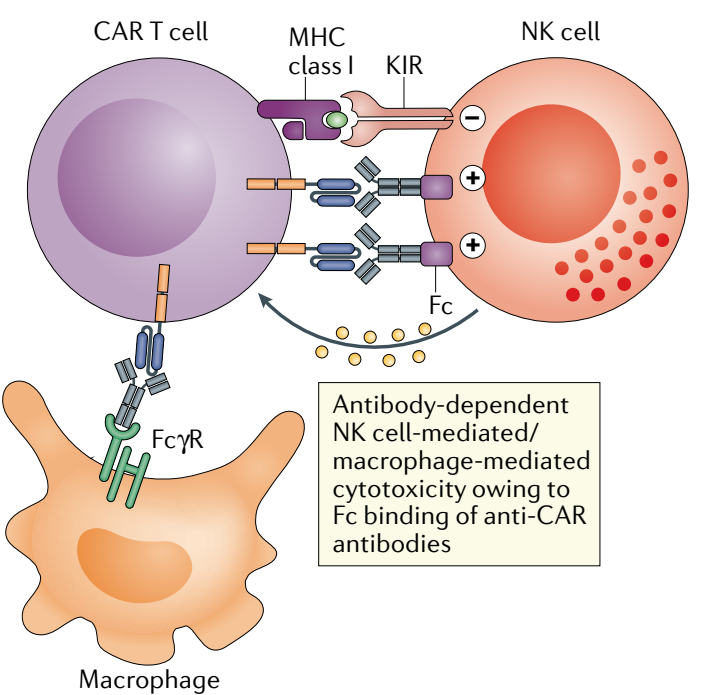

c

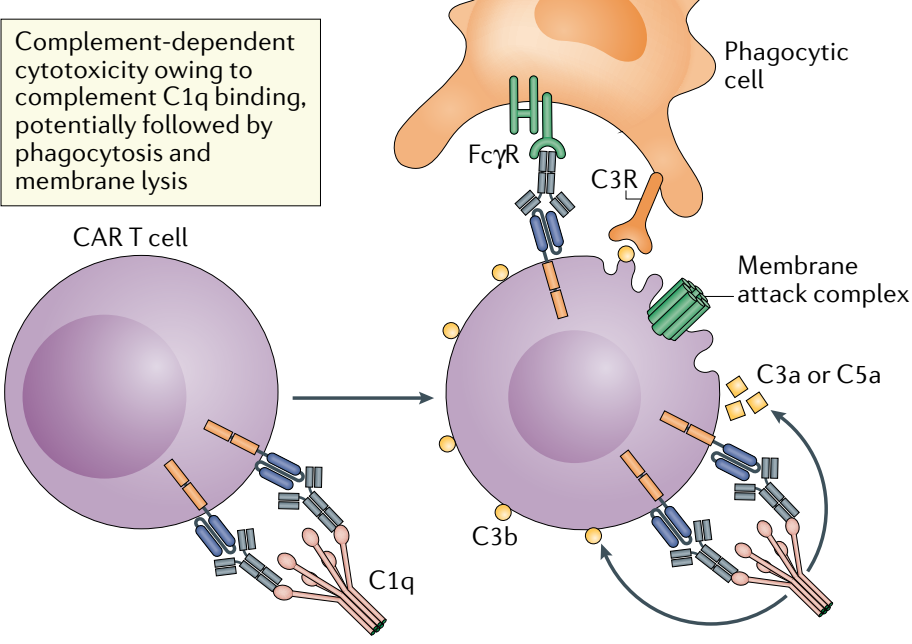

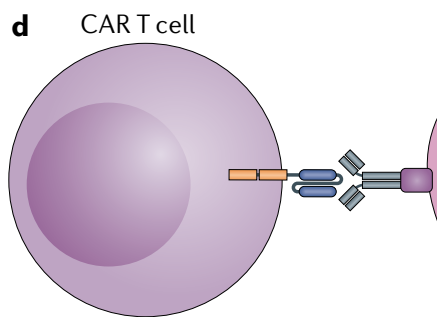

Antibody-targeted mast cell degranulation owing to mast cell binding via the Fc receptor

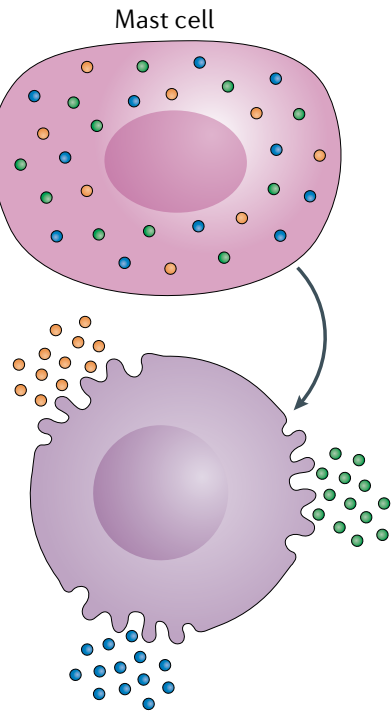

Fig. 1 | Mechanisms of action of anti-CAR immune responses. Acquired anti-chimeric antigen receptor (CAR) immune responses can be humoral or cellular. Cellular immunity probably arises from the processing and cross-presentation of foreign (mouse, viral or non-self human) sequences to the CAR molecule in the context of a major histocompatibility complex (MHC). CAR peptides from apoptotic or necrotic CAR T cells can be displayed by antigen-presenting cells and used to prime $T$ cell responses in secondary lymphoid organs (panel a). CAR-specific $C D 8^{+} T$ cells or cytotoxic $\mathrm{CD}^{+}{ }^{+}$cells ${ }^{153}$ can eliminate CAR T cells that present fragments of the CAR molecule via MHC-mediated recognition. Humoral immunity can be primed through CAR proteins in apoptotic bodies presented by follicular dendritic cells to B cells. Supported by anti-CAR T cells, CAR-specific $B$ cells can expand and then undergo class switching and plasma cell differentiation, producing different classes of immunoglobulins with distinct functions. Anti-CAR antibodies can potentially induce the death of CAR T cells via several mechanisms, including antibody-dependent cellular cytotoxicity, whereby interactions between CAR-bound antibodies and the $F_{c}$ receptor $(F c R)$ domains of innate immune cells, such as natural killer (NK) cells or macrophages, leads to cytotoxicity via the release of perforin and/or granzymes, or phagocytosis (panel b) and complementdependent cytotoxicity owing to CAR-bound antibodies that activate the complement cascade, leading to the formation of membrane attack complexes and cell lysis (panel c). Following CAR engagement, anti-CAR IgE could also bind to the FcR of mast cells, thus promoting degranulation and CAR T cell death (panel $\mathbf{d}$ ). Excessive release of multiple vasoactive mediators in mast cell granula could lead to systemic anaphylaxis, which was lethal in one patient ${ }^{17}$. KIR, killer immunoglobulin-like receptor. 
are noteworthy and could reflect the different assays used for detection. Apparently, the presence of preexisting antibodies or their increase after the infusion of these products was not associated with worse clinical responses. This lack of clinical consequences has also been observed in other studies involving CAR T cells with mouse scFvs targeting CD19 or CD20 (REFS ${ }^{24,25}$ ).

Post-infusion cellular responses to CD19-specific CARs could result in treatment failure. In contrast to pre-existing antibodies, the presence of CAR-specific cytolytic $\mathrm{T}$ cells after infusion has been associated with treatment failure in some but not all clinical trials ${ }^{9,10,26-28}$. The first report of cellular immunity possibly negating therapeutic efficacy involved two patients with follicular lymphoma receiving multiple dose-escalating infusions of first-generation autologous CD19-directed CAR $\mathrm{T}$ cells. These CAR $\mathrm{T}$ cells were produced through an inefficient manufacturing process using $\mathrm{T}$ cell clones with integrated plasmid vectors that required extensive ex vivo culture ${ }^{9}$. The cells were detectable in both patients at 24 hours after the first infusion $\left(10^{8}\right.$ cells $\left./ \mathrm{m}^{2}\right)$, but they failed to persist for even 1 week. Despite re-infusion at higher doses and the use of IL-2 support, the CAR T cells did not survive in vivo. This failure might in part reflect the manufacturing approach but could have been exacerbated by a $\mathrm{T}$ cell-mediated anti-CAR response that existed prior to the first infusion that then expanded after administration. This response was directed towards either the CAR itself or the co-delivered hygromycin phosphotransferase-HSV1-thymidine kinase fusion (HyTK) suicide gene ${ }^{9}$. Similarly, T cell-mediated antiCAR responses have been detected in subsequent trials involving second-generation CD19-directed CAR T cells using mouse-based scFvs and, to a lesser extent, with the use of fully human CAR constructs ${ }^{27,29}$. Notably, intensified lymphodepletion with cyclophosphamide and fludarabine has been identified as a factor that might reduce the extent of anti-CAR cellular immunity ${ }^{27,28}$. Conditioning regimens containing both of these agents are currently considered the standard of care approach prior to initial administration of CD19-targeted CAR T cells ${ }^{30-32}$.

The success of CD19-specific CAR T cell re-infusion might be limited by cellular immunity. Despite the high complete response rates to the first infusion of CD19-directed CAR T cells after lymphodepletion in patients with several haematological malignancies, disease recurrence remains an issue, with approximately $30-50 \%$ of patients having disease relapse within 12 months $^{33}$. Antigen escape through loss of CD19 expression has been seen in 7-25\% of patients, depending on the trial ${ }^{34}$. For those with CD19-positive disease relapse, a repeat infusion of the original CD19-targeted CAR T cell product seems appealing. However, clinical responses to second or subsequent infusions have generally been suboptimal, with complete remissions typically seen in $<25 \%$ of patients ${ }^{35}$. Furthermore, populations of cytotoxic $\mathrm{T}$ cells with specificity towards the CAR have been shown to expand after initial infusion in a subset of patients despite intensified lymphodepletion ${ }^{27}$.
The detection of such cells has been associated with poor expansion of the CAR T cell product after redosing ${ }^{27}$. To date, little is known about which specific epitopes within the CAR construct are recognized by anti-CAR cytotoxic $\mathrm{T}$ cells. The few studies available mostly found T cells with specificity to the mouse scFv FMC63 used in several CD19-specific CARs ${ }^{27,29}$ as well as, to a lesser extent, to other parts of the transgene (such as the signal peptide linker or hinge domains in 3 of 19 patients receiving CD19-specific CAR T cells) ${ }^{29}$ (Supplementary Table 1).

Immunogenicity in haematological indications beyond CD19 CAR T cells. Another promising CAR T cell candidate currently in clinical development targets plasma cell malignancies. Specifically, CAR T cells redirected to recognize $B$ cell maturation antigen (BCMA) have induced potent antitumour responses in patients with advanced-stage multiple myeloma in seven large clinical cohorts (as of July 2020), although rates of complete remission are thus far lower and responses are less durable than with CD19-targeted CAR T cells in patients with B-ALL or $\mathrm{NHL}^{36-38}$. All trials used lymphodepletion in the majority of patients (with cyclophosphamide or fludarabine and cyclophosphamide), and only one study provided data on CAR immunogenicity ${ }^{36-42}$. In a recent approach described by Xu et al. ${ }^{40}$, a bispecific CAR targeting two distinct BCMA epitopes using two camelderived antigen-binding domains combined within a single CAR construct induced the highest yet reported complete remission rate of $76 \%(13 / 17)$ in patients with multiple myeloma. Anti-CAR antibodies were detected in all patients with disease relapse after an initial complete remission and correlated with a decrease in circulating CAR T cell numbers. Thus, humoral immunity could be associated with CAR $\mathrm{T}$ cell inactivation and treatment failure in patients receiving products that do not deplete endogenous B cells. Indeed, plasma cell depletion by BCMA-directed CAR T cells might be insufficient to blunt the early $\mathrm{B}$ cell responses that drive humoral immunity towards CARs (FIG. 2).

Several novel CAR T cell approaches are in development for other indications such as $\mathrm{T}$ cell malignancies (NCT03590574) ${ }^{43}$. Introducing CAR constructs with specificity for pan $\mathrm{T}$ cell-lineage antigens has thus far proved challenging owing to CAR T cell fratricide, a phenomenon involving self-killing among CAR T cells ${ }^{44,45}$. This process could both limit effective manufacturing of the product and reduce persistence of the cells in vivo. An exception to these limitations is provided by the development of anti-CD5 CAR T cells ${ }^{46}$, in which a subpopulation of CD5-specific CAR T cells downregulates endogenous CD5 expression, thus avoiding fratricide and enabling expansion for clinical application. Early reports from an ongoing phase I trial suggest efficacy of such CD5-targeted CAR T cells in patients with T cell acute lymphoblastic leukaemia or $\mathrm{T}$ cell lymphoma, without $\mathrm{T}$ cell aplasia ${ }^{47}$. The continued depletion of endogenous $\mathrm{T}$ cells could theoretically impede adaptive immune responses towards tumours and pathogens but might also limit the extent of anti-CAR immunity, thus improving CAR T cell persistence. 
a

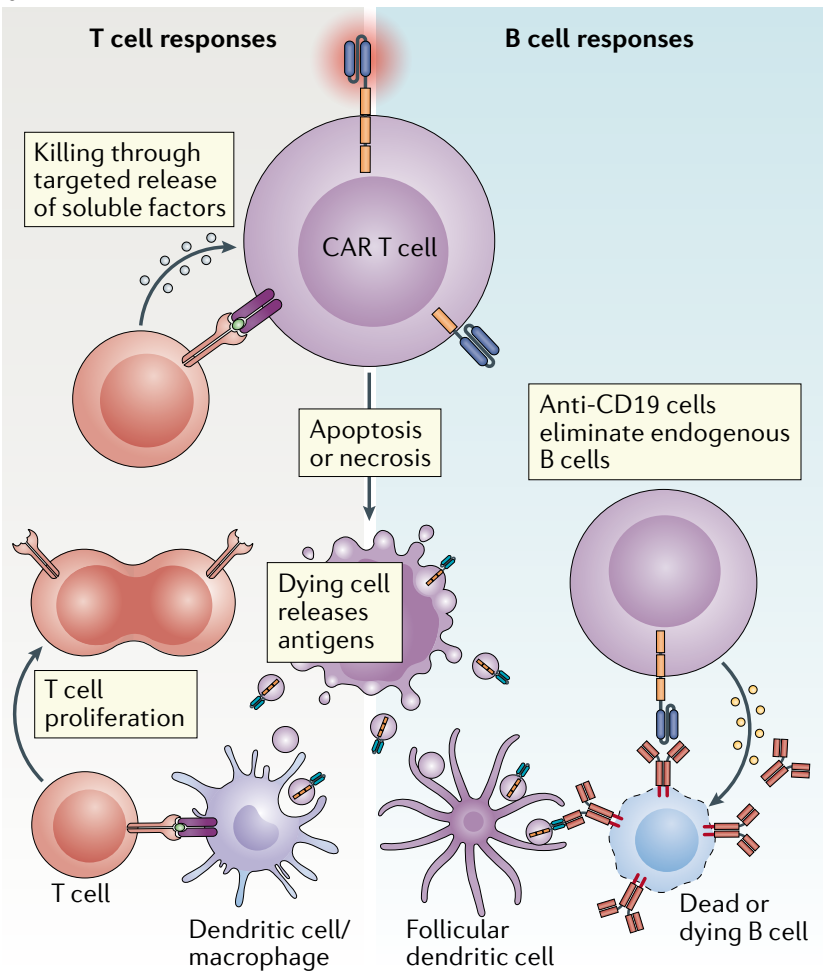

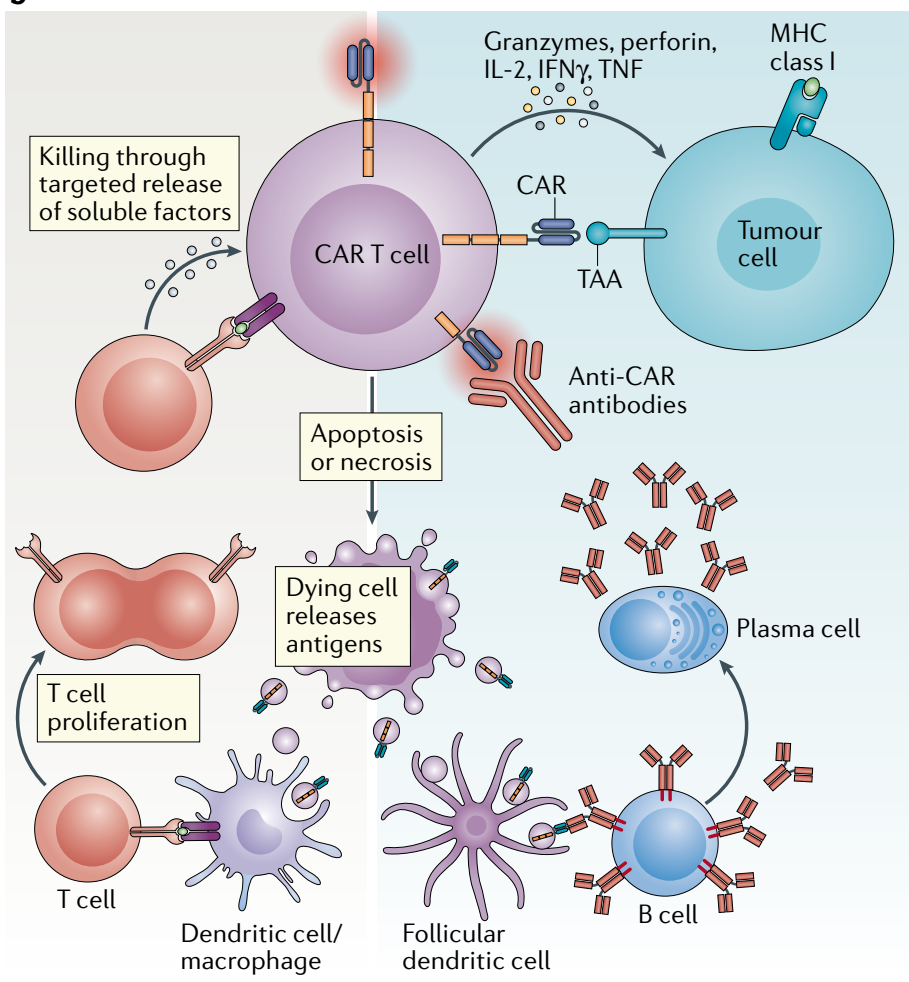

Fig. 2 | Differences in anti-CAR immune responses following the targeting of $B$ cell versus solid-tumour antigens. a | Chimeric antigen receptor (CAR) T cells targeting B cell malignancies induce aplasia of non-malignant B cells, thus reducing the potential for anti-CAR humoral immunity. $\mathbf{b}$ |CAR T cells directed to solid-tumour antigens (targets not expressed on B cells) might have an increased risk of inducing both cellular and humoral anti-CAR immunity. MHC, major histocompatibility complex; TAA, tumour-associated antigen.

Early trials of CAR T cell therapy in solid tumours revealed anti-CAR immunity. Eliciting clinical responses with CAR T cells in patients with advanced-stage solid tumours has also proved challenging, in part owing to poor $\mathrm{T}$ cell infiltration of the tumours and/or limited CAR T cell persistence ${ }^{7,8}$, which have been linked, in some cases, to anti-CAR immune responses ${ }^{48,49}$ (FIG. 2). For example, Kershaw et al. ${ }^{16}$ conducted a phase I trial to evaluate the safety of adoptive immunotherapy with autologous first-generation CAR T cells targeting the $\alpha$-folate receptor (FRa) in patients with metastatic ovarian cancer. These investigators observed an antibody-mediated immune response to the CAR $\mathrm{T}$ cells that inhibited both IFN $\gamma$ release (an indicator of anti-FR $\alpha$ activity) and cytotoxic activity against the FRa-positive tumour cells. This response substantially reduced the efficacy of the CAR T cells and might have led to their rapid clearance, as observed in the trial ${ }^{16}$.

Hege et al. ${ }^{48}$ reported outcomes from the first two clinical trials (conducted in the 1990s) evaluating CAR $\mathrm{T}$ cells in patients with solid tumours, with a focus on persistence and immunogenicity. These first-generation CAR T cell products contained a humanized antibody (huCC49) directed towards a tumour-associated glycoprotein (TAG-72) and elicited an anti-idiotype immune response via the formation of anti-CAR antibodies to the mouse-derived TAG-72-binding domain of huCC49. This immune response led to the elimination of the infused TAG-72 CAR T cells in $<14$ weeks, despite repeated high-dose infusions of CAR T cells $\left(1 \times 10^{10}\right)$ in the majority of patients ${ }^{48}$. A preceding study using the fully mouse-derived variant of CC49 revealed that the antibody itself might have antigen-binding epitopes with particularly high immunogenic potential, resulting in HAMA responses in the majority of patients $(54 \%)^{13}$. Follow-up investigations led to next-generation huCC49 humanized constructs bearing only the specificitydetermining residues of the mouse TAG-72-binding epitopes grafted onto human antibodies ${ }^{50}$. These early trials demonstrate the importance of minimizing the inclusion of mouse components when designing CAR $\mathrm{T}$ cell products for solid tumours.

\section{Profiling the immunogenicity of next-generation} CARs in solid tumours. Early studies involving firstgeneration CAR constructs helped to elucidate the likelihood of immunogenic responses in patients with solid tumours ${ }^{16,48,49}$. However, the limited efficacy of these CAR T cells prompted a shift towards the use of second-generation CARs after improved antitumour responses were observed in preclinical models and in patients with haematological malignancies ${ }^{51-55}$. For example, Ahmed et al. ${ }^{56,57}$ used autologous T cells expressing a second-generation CAR based on the mouse HER2-specific monoclonal antibody FRP5. In one trial, FRP5-scFv HER2-exodomain-specific CAR T cells were administered to 19 patients with advancedstage HER2-positive sarcomas. These investigators 
observed low but detectable levels of HER2-specific CAR transgenes in peripheral blood for up to 2 years after repeat infusions ${ }^{56}$. Similar CAR T cell kinetics were observed in another clinical study conducted by the same group involving patients with progressive glioblastoma ${ }^{57}$. Despite these as well as other trials showing unsatisfactory clinical responses ${ }^{56-59}$, the role of cellular and humoral anti-CAR responses was not intensely investigated as a factor that may have contributed to treatment failure.

The aforementioned studies, which involved patients with solid tumours, did not include lymphodepletion prior to CAR T cell infusion. In contrast, in a case report published in July 2020, three cycles of lymphodepletion followed by infusions of second-generation CAR T cells targeting HER2 enabled the peripheral expansion and bioavailability of the infused cells ${ }^{60}$. This approach resulted in a complete response in a child with metastatic rhabdomyosarcoma, which was then consolidated with repeat CAR T cell infusions without further lymphodepletion but with an anti-PD-1 antibody. Longitudinal immune monitoring in this child revealed T cell-repertoire remodelling with immunodominant clones and serum autoantibodies reactive to oncogenic pathway proteins. Despite an evident strong endogenous immune response, the patient remained HAMA-negative even after receiving a total of 15 infusions of a CAR T cell product containing a mouse scFv. Drawing definitive conclusions based on observations from a single patient is difficult; therefore, further investigations are warranted to better elucidate the anti-CAR immune responses and their potential interplay with lymphodepletion, particularly where redosing is required. Ultimately, mitigating immunogenicity using intensified lymphodepletion might contribute to improving CAR T cell engraftment in patients with solid tumours ${ }^{61-63}$.

\section{Reducing inherent CAR immunogenicity}

Several components of CAR constructs have the potential to trigger anti-CAR immune responses in patients. Various approaches can be used to render these components less immunogenic.

Humanizing tumour-reactive scFvs. Several investigators are developing and testing humanized scFvs to circumvent the anti-CAR responses associated with mouse-derived scFvs. Humanized constructs are likely to be less immunogenic and might also be preferable for salvage therapy in patients with disease relapse after initial mouse-derived CAR T cell infusion (FIG. 3) or as an option for CAR T cell-naive patients ${ }^{29,50,64-66}$. The first treatment of patients with B cell NHL using a fully human CD19-directed CAR induced fewer CAR-specific $\mathrm{T}$ cell responses compared with a similar cohort who received CAR $\mathrm{T}$ cells containing a mouse-derived $\mathrm{scFv}^{29}$. Another strategy is to substitute traditional scFvs with immunoglobulin heavy-chainonly recognition domains that lack light chains and potentially immunogenic linker sequences (such as heavy-to-light chains or other associated junctions) $)^{67}$. These heavy-chain CAR constructs have shown robust target affinity and efficacy in preclinical models ${ }^{68-70}$. The first clinical proof-of-efficacy of a BCMA-specific

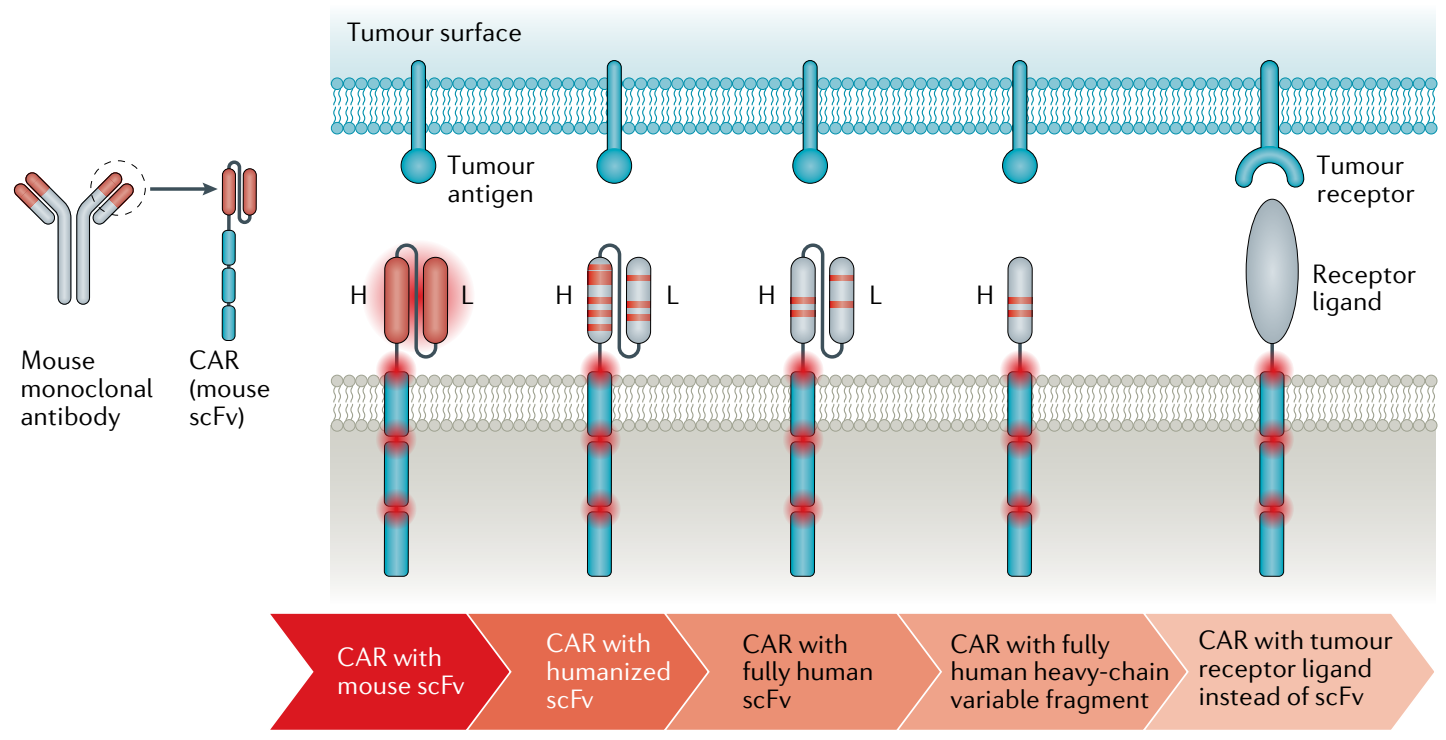

Fig. 3 | Engineering CAR T cells to reduce their inherent immunogenicity. Improving chimeric antigen receptor (CAR) design in order to limit inherent immunogenicity involves selecting tumour-binding moieties with the least immunogenic potential. Here, we show methods of replacing elements of single-chain variable fragments (scFvs) derived from mouse antibodies with human sequences or replacing the entire mouse-derived scFv with human-derived heavy-chain variable fragments or fully human scFvs. CARs with heavy-chain-only variable fragment tumour-recognition domains have the additional advantage of not requiring linkers, which are another possible source of immunogenicity. As an alternative to scFvs, the ligand of a tumour-associated receptor (or vice versa) can be attached to the constant regions of the CAR. Such constructs exploiting interactions between endogenous receptors and their ligands might enable tumour targeting with minimal risk of immunogenicity. 
fully human heavy-chain-only construct was reported in November 2019 (REF. ${ }^{71}$ ). Theoretically, constructs derived solely from human protein components could still initiate immune responses involving anti-idiotype antibodies in patients ${ }^{48,72}$. Therefore, head-to-head clinical trials directly comparing the immunogenicity of mouse-derived and humanized scFvs are warranted.

Exchanging scFvs with alternative tumour-specific domains. Aside from scFv-based CARs, tumour cells can also be targeted via endogenous tumour-specific receptor-ligand interactions. The $s c F v$ portion of the CAR can be replaced either by the extracellular domain of a receptor or by a ligand that binds to receptors overexpressed by tumours. Moieties currently in preclinical and early clinical evaluation include chimeric NKG2D receptors ${ }^{73-75}$, membraned-tethered IL-13-cytokine CARs targeting IL-13Ra2-expressing tumour cells ${ }^{76-78}$, integrin $\alpha_{\mathrm{v}} \beta_{6}$-binding peptides ${ }^{79,80}$ and heregulin- $\zeta$ chimaeras $^{81}$. Such tumour-specific receptor-ligand interactions are expected to be less immunogenic than traditional scFvs because they have human peptide sequences and are therefore likely to be recognized as self-proteins. Furthermore, universal CAR designs with extracellular domains that can be loaded with tumourtargeting antibodies in vivo might enable renewed redirection of CAR $\mathrm{T}$ cell specificity in the event of antigen escape $^{82,83}$. However, no immunogenicity data are available thus far regarding the use of such universal receptor strategies.

Mutating CAR spacers. Optimal engagement of the scFv with the target epitope is paramount for effective CAR $\mathrm{T}$ cell therapy. In particular, proximal epitopes located close to the tumour cell membrane might require CAR $\mathrm{T}$ cells with longer and more flexible hinge domains. Many groups have used the constant regions of various IgG heavy chains as hinges ${ }^{84,85}$. In their original form, the $\mathrm{CH} 2$ and $\mathrm{CH} 3$ domains within these IgG-derived spacers could be targeted by Fc-receptors on innate immune cells, such as macrophages, granulocytes and natural killer (NK) cells, and might pose an immunogenicity risk (FIG. 1). Indeed, the presence of an Fc-binding spacer containing the $\mathrm{CH} 2$ domain might have contributed to the poor persistence of CD19-targeted CAR T cells reported by Jensen et al. as well as in other clinical studies ${ }^{9,25,86}$. This phenomenon can be avoided by mutating the Fc receptor-binding epitopes within the spacer, thereby preventing innate immune cell activation ${ }^{87,88}$.

\section{Immunogenicity of novel CAR technologies}

Cytokine self-support. Cytokine support technologies designed to enhance the antitumour efficacy of CAR T cells, particularly with the goal of overcoming cytokine starvation in tumours with immunosuppressive microenvironments, are an area of extensive preclinical and clinical investigation ${ }^{7,89}$. The systemic administration of cytokines, such as IL-15, increases the risk of immunogenic responses in patients and can cause toxicities such as fever, rigors, hypotension, thrombocytopenia and lymphocytopenia, among others ${ }^{90}$. Therefore, other approaches using engineered CAR T cells to locally release soluble cytokines have been used, demonstrating potent activity in preclinical models ${ }^{91-93}$. Bystander T cell activation is a theoretical risk inherent to such paracrine cytokine support strategies ${ }^{94,95}$. Early clinical results with this approach are encouraging ${ }^{96}$, although whether paracrine cytokine support might amplify any anti-CAR responses remains to be elucidated. Alternatively, a constitutively active cytokine receptor or a cytokine receptor signalling domain within the CAR molecule can provide immunomodulatory cytokine signalling solely to the CAR T cells without affecting bystander lymphocytes ${ }^{97,98}$ and this strategy is currently being investigated in clinical trials (NCT03635632 and NCT04099797).

Suicide genes and elimination markers. Despite their effectiveness in patients with certain haematological malignancies, CAR T cells also pose safety risks due to on-target, off-tumour toxicities and/or the unpredictable adverse effects of add-on technologies. Suicide genes and elimination markers have been investigated as strategies that can be co-introduced alongside the CAR transgene to enable the targeted depletion of gene-modified cell products upon the emergence of severe adverse event $^{11,99,100}$. However, such approaches are not without a risk of immunological complications. For example, Berger et al. ${ }^{99}$ identified multiple immunogenic epitopes in $\mathrm{T}$ cells expressing herpes simplex virus thymidine kinase (HSV-TK) as an inducible suicide gene, resulting in rapidly evolving anti-CAR $\mathrm{T}$ cell responses mediated by $\mathrm{CD} 4^{+} \mathrm{T}$ cells and $\mathrm{CD} 8^{+} \mathrm{T}$ cells. Thus, the HSV-TK suicide gene might also have contributed to the rejection of CD19-directed CAR T cells observed in the trial conducted by Jensen et al. ${ }^{9}$, as mentioned earlier.

Safety systems adapted from proteins of human origin (such as surface-bound elimination markers, including truncated EGFRs or the iCaspase 9 suicide system) avoid introducing xenogeneic components that might increase the risk of immunogenicity ${ }^{100-102}$. To implement safety genes in CAR T cell products, many researchers choose to co-express the CAR construct and a suicide switch on a single polycistronic construct separated by an internal ribosome entry site or a viral 2A sequence that contains self-cleaving peptides. Virus-derived 2A sequences have not been shown to be immunogenic in ex vivo studies ${ }^{103}$, although only prospective clinical trials can definitively address the issue of their immunogenicity in patients. Notably, research published in 2000 indicates that internal ribosome entry site insertion might reduce the expression level of downstream transgenes such as the suicide switch ${ }^{104}$. More recently, gene editing has been used to create transgene-free suicide switches that avoid the risk of transgene-related immunogenicity, although this approach currently requires further investigation ${ }^{105}$.

Gene editing with transiently delivered nucleases. Programmable nucleases provide a method of enhancing CAR T cell functionality via gene editing. This approach has been used to disrupt the expression of endogenous immune-checkpoint proteins and thus boost the performance of CAR T cells ${ }^{106-108}$ and, potentially, to enable their co-administration with therapeutic monoclonal 
antibodies $^{109}$. Furthermore, gene editing might limit the extent of fratricide owing to reduced cell-surface antigen expression, enabling clinical investigations of CAR $\mathrm{T}$ cells for common $\mathrm{T}$ cell antigens beyond CD5 (such as CD3 and CD7 ${ }^{44,45}$. Importantly, gene editing could also be used to evade $\mathrm{T}$ cell-mediated anti-CAR immunity by eliminating cell-surface MHC expression as has been demonstrated in vitro and in vivo using allogeneic CAR T cells ${ }^{106,110,111}$ (FIG. 4).

Aside from unintended off-target gene editing events, the induction of host immune responses combined with pre-existing humoral and cellular immunity has been identified as a risk factor associated with the CRISPR-Cas9 genome editing system ${ }^{112-117}$ that could lead to the T cell-mediated elimination of Cas9-expressing cells ${ }^{117,118}$. Nevertheless, Stadtmauer et al. ${ }^{108}$ demonstrated that the temporary delivery of CRISPR-Cas9 components as ribonucleoprotein complexes did not affect the persistence of autologous TCR-redirected $\mathrm{T}$ cells in three patients. The rapid degradation of the immunogenic protein as well as dilution during ex vivo expansion probably explain these results. Thus, transient delivery, as opposed to constitutive overexpression, could be the preferred method of CAR T cell gene editing in cells with intact antigen processing and warrants further investigation.

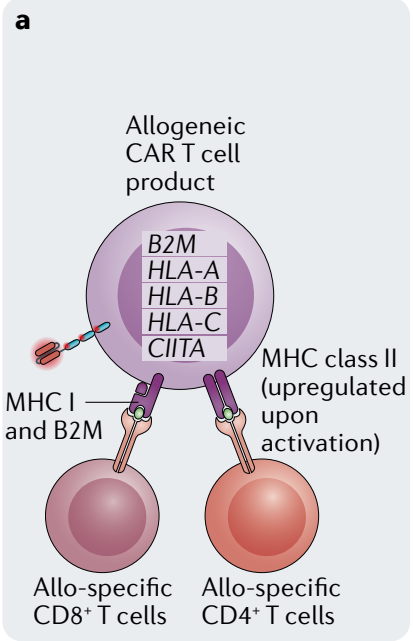

e

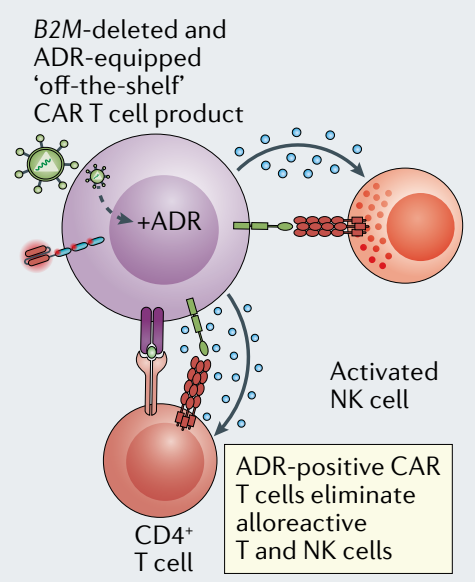

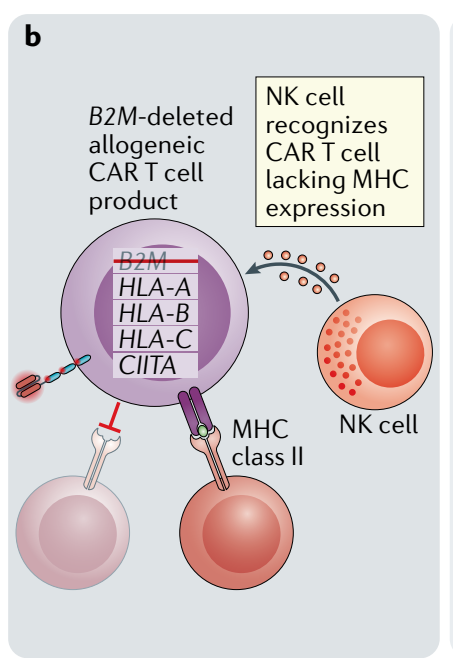

f

B2M-edited with HLA-E enhanced 'off-the-shelf' CAR T cell product

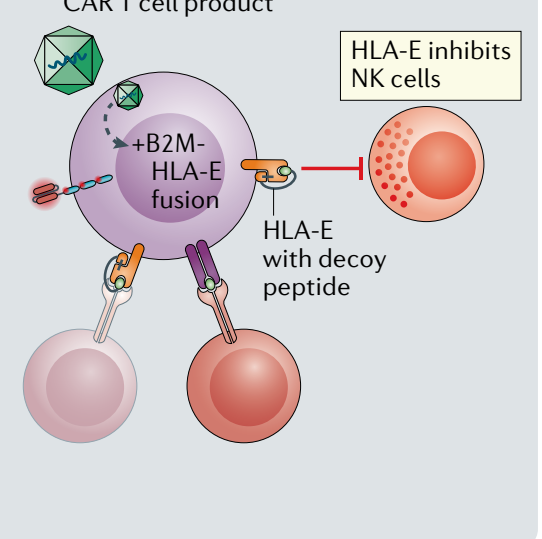

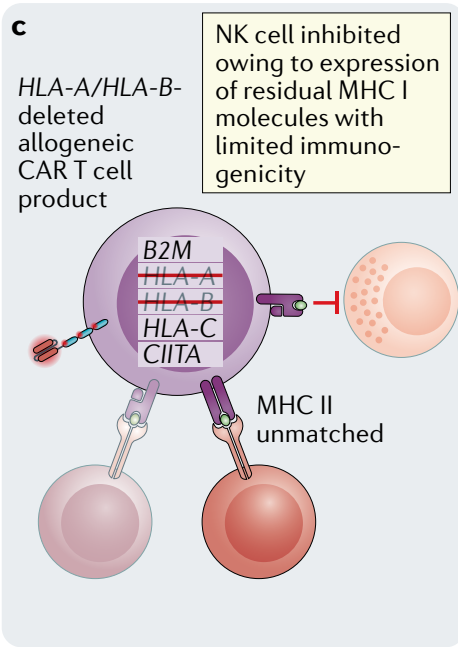

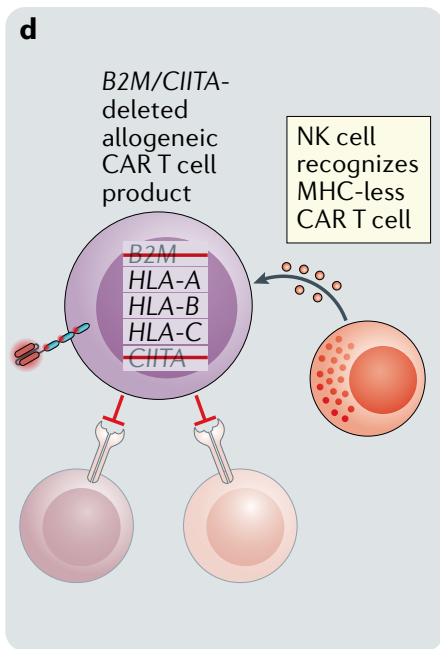

$\mathbf{g}$

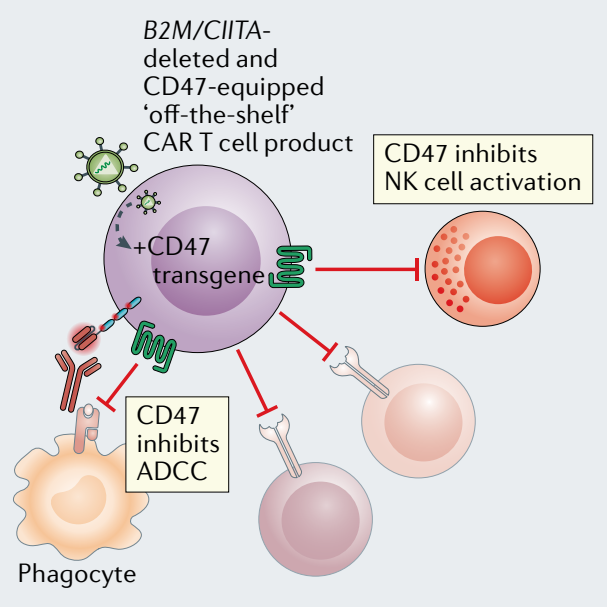

Fig. 4 | Strategies to avoid immune elimination of allogeneic CAR T cells. a | The presence of incompatible major histocompatibility complexes (MHCs) can boost anti-chimeric antigen receptor (CAR) responses to allogeneic CAR T cells. Resting T cells express MHC class I (MHC I), while T cells can also upregulate MHC class II (MHC II) after activation. b | MHC acts as a potent inhibitory receptor via the killer immunoglobulin-like receptor (KIR) on natural killer (NK) cells. Eliminating MHC I from the surface of resting CAR $T$ cells can prevent alloimmune reactions while also increasing the risk of NK cell-mediated cytotoxicity. c | Multiplex gene editing can be used to eliminate CAR T cell-surface MHC expression while also sparing the less-diverse human leukocyte antigen (HLA)-C subtype, which might minimize the risk of rejection by NK cells. Careful MHC matching of donor-derived products should also be performed. $\mathbf{d}$ | Disrupting the functional expression of CIITA, which encodes the master transcriptional regulator of $\mathrm{MHC} \mathrm{II,} \mathrm{or} \mathrm{of} \mathrm{the} \mathrm{common} \mathrm{constant} \mathrm{chain} \mathrm{CD74} \mathrm{could} \mathrm{be} \mathrm{used}$ to remove MHC II from the cell surface without provoking alloimmune responses to activated CAR T cells. e | Alloimmune defence receptors (ADRs) targeting the activation marker 4-1BB on allospecific T cells might improve the persistence of allogeneic CAR T cells. ADRs introduced into $B 2 M$-negative and $\mathrm{MHCl}$-deficient CAR T cells are likely to actively eliminate the risk of NK cell lysis. $\mathbf{f} \mid$ Site-specific insertion of universal $\mathrm{MHC}$ I constructs (such as those based on a decoy peptide-loaded monomorphic HLA-E) into the B2M gene prevent NK cell targeting of allogeneic-induced pluripotent stem-derived cells. $\mathbf{g}$ Alternatively, the overexpression of CD47 prevents effective alloreactive T cell/NK cell activation and antibody-dependent cellular cytotoxicity (ADCC) by acting as a 'do not eat me' signal. 


\section{Immunity and allogeneic CAR T cells}

Off-the-shelf allogeneic CAR T cell products created using cells from healthy donors are an attractive alternative to the autologous approach and could potentially broaden the clinical applicability of these cells and ensure timely availability ${ }^{119}$. However, non-self MHCs expressed on these CAR T cells could elicit host-derived immune responses that lead to immune-mediated elimination of the product. Allogeneic CAR T cells could be targeted by the patient's alloreactive T cells with specificity to foreign major MHC antigens ${ }^{120,121}$. Even with partially human leukocyte antigen (HLA)-matched donors, minor antigen differences owing to polymorphisms could create additional immunogenic epitopes ${ }^{122}$. Indeed, data published in December 2020 (REF. ${ }^{123}$ ) indicate that preconditioning with chemotherapy plus the anti-CD52 antibody alemtuzumab, which depletes both $\mathrm{B}$ cells and $\mathrm{T}$ cells, is required for the short-term antitumour activity of allogeneic CD19-specific TCR-edited T cells in both adult and paediatric patients with treatment-refractory B-ALL. Importantly, alemtuzumab would also diminish CD52-expressing CAR T cells after infusion; therefore, CD52 was deleted in the CAR T cells used in this trial. Despite the addition of alemtuzumab to lymphodepletion, the allogeneic CAR T cells displayed limited persistence after endogenous $\mathrm{T}$ cell reconstitution ${ }^{123}$. These results highlight the need for strategies to overcome the barriers to allo-reactive immunity created by even minor HLA mismatches.

Several approaches have been used to generate CAR $\mathrm{T}$ cells with reduced expression of MHC antigens, thus enabling the treatment of patients regardless of HLA disparities (FIG. 4). The elimination of MHC class I (MHC I) by direct editing of the HLA locus or by disruption of the gene encoding $\beta 2$-microglobulin (B2M), which is required for MHC I expression, have the potential to prevent allo-rejection by $\mathrm{CD}^{+} \mathrm{T}$ cells ${ }^{106,110}$. However, because $\mathrm{T}$ cell activation causes the upregulation of MHC II, allospecific $\mathrm{CD} 4^{+} \mathrm{T}$ cell-mediated CAR T cell rejection could also occur. The elimination of MHC II expression via the deletion of CIITA, the master transcription factor for MHC II genes, could mitigate this rejection ${ }^{111}$.

These approaches offer some promise against $\mathrm{T}$ cell-mediated rejection; however, paradoxically, the loss of HLA molecules renders allogeneic CAR T cells vulnerable to NK cell killing. Therefore, NK cell inhibitory transgenes have been developed to promote the persistence of genetically modified cells in immunocompetent hosts (FIG. 4). For example, Gornalusse et al. ${ }^{124}$ developed cells that are resistant to rejection by both $\mathrm{CD}^{+} \mathrm{T}$ cells and NK cells by expressing the non-polymorphic HLA-E molecule fused to B2M and a decoy antigen in human pluripotent stem cells. Other strategies, for example, involving multiplex gene editing, might be used to eliminate highly polymorphic $H L A-A$ and $H L A-B$ alleles while sparing the less diverse $H L A-C$, thus retaining an important endogenous NK cell-inhibitory receptor ${ }^{125}$. Alternatively, the overexpression of CD47 can inhibit alloreactive T cells and NK cell activation as well as providing a 'do not eat me' signal to phagocytes that prevents antibody-dependent cellular cytotoxicity ${ }^{126,127}$. In July 2020, Mo et al. ${ }^{128}$ published a report describing an 'alloimmune defence receptor' that enables CAR T cell-mediated killing of alloreactive $\mathrm{T}$ cells and NK cells through recognition of the activation marker 4-1BB in preclinical models without the need for genetic modifications of the HLA system. Intriguingly, the expression of alloimmune defence receptors by engineered immune cells compromised neither allogeneic CAR T cell expansion nor the antitumour immune response in mouse models ${ }^{128}$.

Extensive lymphodepletion can also reduce the risk of immediate allorejection $^{129,130}$. The downside of this approach is the potential of graft-versus-host disease (GvHD) caused by persistent allogeneic CAR T cells or the proliferation of endogenous non-CAR donor T cells retaining alloreactive TCR expression. To mitigate the risk of GvHD, investigators have transferred CAR transgenes into virus-specific T cells, thus generating safer products because the TCRs of such cells are less likely to be alloreactive ${ }^{57,131-134}$. Alternatively, CARs can be introduced into non-conventional $\mathrm{T}$ cells with restricted TCR repertoires (such as $\gamma \delta \mathrm{T}$ cells, mucosa-associated invariant T cells, NK T cells and invariant NK T cells), as these all pose a lesser risk of $\mathrm{GvHD}^{135-137}$. As a further alternative, the TCRs of allogeneic CAR T cells could be disrupted using gene editing ${ }^{109}$.

\section{Anti-CAR immune responses in the clinic}

Longitudinal investigations of anti-CAR immune responses would be particularly important in understanding how immunogenicity can lead to treatment failure. Current professional society guidelines for the use of CD19-directed CAR T cells do not include recommendations regarding the evaluation or clinical management of suspected anti-CAR immunity ${ }^{32,138-140}$. In this section, we outline the need to develop validated assays to adequately monitor the immunogenicity of CAR T cell candidates and enable investigators to assess the clinical relevance of these responses and, if appropriate, implement suitable evidence-based mitigation and management strategies (FIG. 5).

\section{Monitoring anti-CAR immunity in clinical trials and} clinical practice. The observation of lethal anaphylaxis attributed to HAMA after infusion of mesothelinspecific CAR T cells ${ }^{17}$ might have prompted regulatory agencies to recommend addressing the risk of immunogenicity in phase I safety trials if an immunological reaction is suspected (FIG. 5). Several assays have been designed in various clinical trials to test for humoral or cellular immune responses to CAR T cells (BOX 1). Beyond HAMA, however, these assays are rarely certified according to Clinical Laboratory Improvement Amendments and are currently not widely available. At the same time, HAMA is, by definition, only of value for the detection of antibodies to mouse-derived scFvs. Particularly subsequent infusions of mouse-derived CAR T cells could be preceded by HAMA evaluation, which might help to elucidate possible mechanisms of primary resistance and/or limited responses to second or subsequent infusions ${ }^{141}$. As novel constructs are humanized, HAMA assessments might become less 


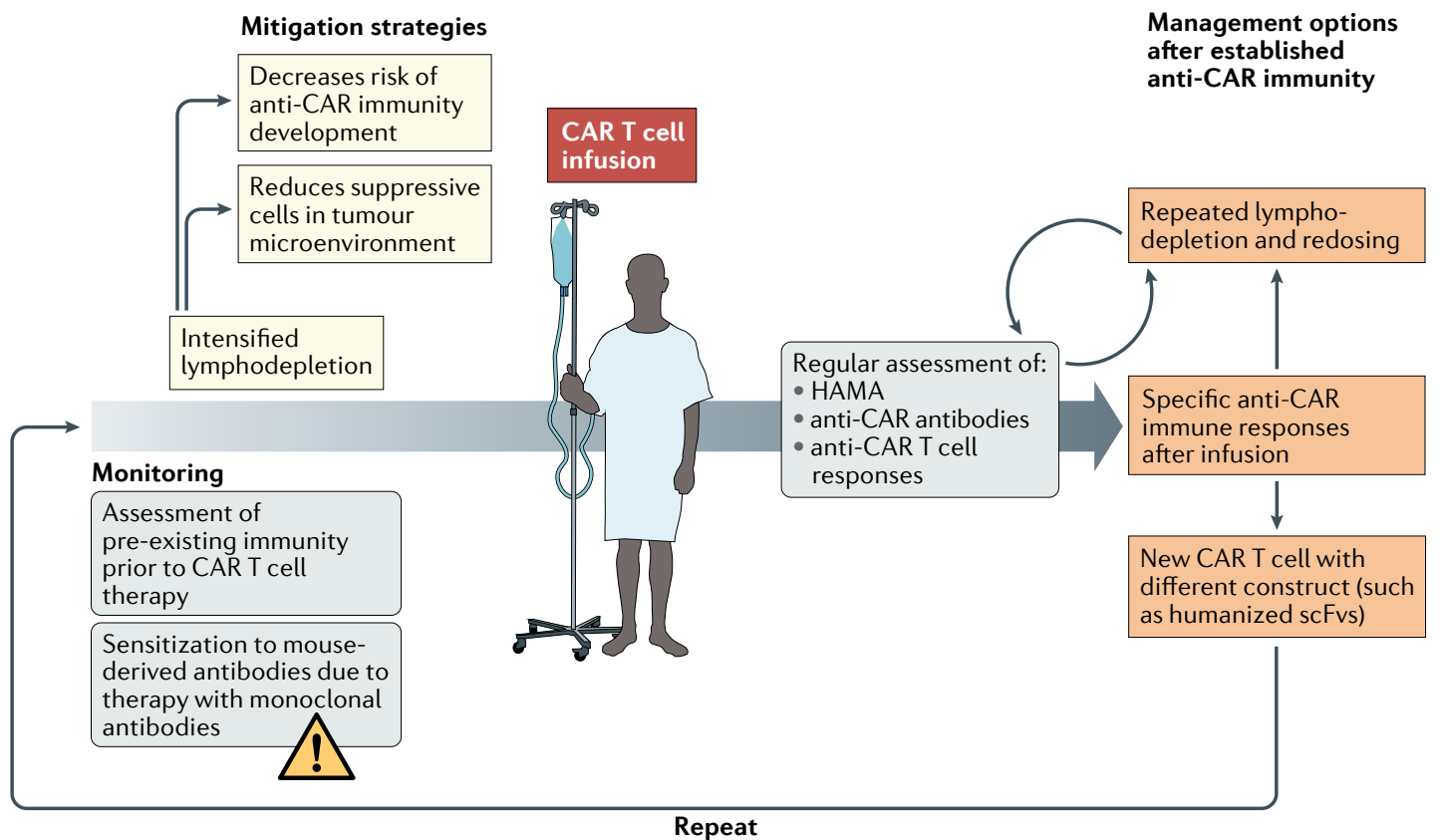

Fig. 5 | Monitoring, mitigation and management of anti-CAR immunity in the clinic. Mitigation of anti-CAR immunity through lymphodepletion regimens can reduce the numbers of circulating lymphoid cells as well as of antigen-presenting cells prior to CAR T cell infusion. Furthermore, cytoreductive treatments might reduce the numbers of certain immunosuppressive cells in the tumour microenvironment, leading to tumour cell death and creating a pro-inflammatory milieu that promotes the antitumour efficacy of CAR T cells ${ }^{154}$. The monitoring of patients receiving CAR T cells should begin as soon as a patient is considered for therapy. Conventional cancer treatment regimens, including monoclonal antibodies of mouse origin ${ }^{155}$, can induce human anti-mouse antibodies (HAMAs) in patients ${ }^{12,13}$. Therefore, careful evaluation of preexisting immunity is important for the avoidance of anaphylaxis after CAR T cell infusion ${ }^{17}$. Subsequently, monitoring in clinical trials should include surveillance for possible HAMAs and specific anti-CAR immune responses, including antibody and T cell analysis. The management of low-level anti-CAR immunity can be achieved using repeat lymphodepletion to reduce anti-CAR immunity prior to redosing with the same CAR T cell product. Humanized CAR T cell constructs could be considered in patients who progressed or relapsed after a previous infusion of CAR T cells containing mouse single-chain variable fragments (scFvs) or who required subsequent infusions to prevent disease progression. However, this approach could be associated with additional costs and/or treatment delays.

relevant and developing new assays, both for humanized constructs and potentially for those derived from non-mouse, non-human sources, will be a growing need. Until such companion assays are available, investigators could consider cryopreserving serum samples and peripheral blood mononuclear cells collected at multiple time points for later analysis.

Very few clinical studies involving patients with solid tumours have investigated anti-CAR antibody responses beyond HAMAs, despite reports indicating potent immune responses to non-human CAR sequences and mutant oncogenic proteins ${ }^{49,60}$. Given that CAR T cell expansion and persistence remain suboptimal in trials for solid tumours, the role of immune responses to the CAR constructs used is becoming more relevant and should be revisited. Thus, establishing validated assays for the characterization of humoral and cellular immune responses to CAR constructs and the associated transgenes is of paramount importance. Assays designed to detect anti-CAR antibodies can be cell-based or enzymelinked immunosorbent assay (ELISA)-based and should ideally be able to measure the immunogenicity of all CAR components expressed on the T cell surface and predict their likely effects on the cell product (such as cytotoxic potential) ${ }^{142}$ (BOX 1). For cellular immune responses, assays that enable mapping of the most immunogenic epitopes within the CAR constructs are most likely to inform better CAR design and the development of novel technologies that improve efficacy. Overall, these efforts could also benefit from the implementation of common terminologies and the lessons learned from the assessment and reporting of immunogenicity for biologics (such as therapeutic proteins) $)^{143-146}$.

Mitigating immunogenicity with intensified lymphodepletion. Lymphodepleting conditioning regimens administered prior to CAR T cell therapy are expected to affect not only the lymphoid compartment (including $B$ cells and T cells) but also myeloid cells. The transient removal of professional antigen-presenting cells could reduce the likelihood of antigen cross-presentation when CAR T cells are expanding in the circulation and within lymphoid tissues. Cyclophosphamide-based chemotherapy is also known to deplete regulatory $\mathrm{T}$ cells ${ }^{147}$, which can otherwise limit both the antitumour efficacy and persistence of CAR T cells. Most investigators routinely incorporate fludarabine/cyclophosphamide-based lymphodepletion to improve treatment outcomes and overcome the possibility of immunological rejection with initial CAR T cell infusions ${ }^{27,28,32}$. Intensifying this 
regimen is also being considered for subsequent infusions. Interestingly, Gauthier et al. ${ }^{35}$ demonstrated that intensified lymphodepletion (with fludarabine plus cyclophosphamide versus cyclophosphamide alone) prior to the first CAR infusion is associated with improved responses to a second, higher dose of CD19-targeted CAR T cells in a subset of patients.

Managing relapsed disease with humanized CARs and other constructs. To avoid premature CAR T cell inactivation resulting from immune responses to the mouse-derived scFvs, alternative CAR T cell products using humanized scFvs can be considered. Early studies involving humanized CD19-targeted CAR T cell constructs reported varied clinical response rates, including in patients who progressed or relapsed after a previous infusion of CAR T cells containing mouse scFvs. For example, Maude et al. ${ }^{64,148}$ infused 16 patients with B-ALL who either did not respond to or had disease progression on CTL019 CAR T cells (which include the mouse-derived FMC63 scFv) with an alternative CD19-specific CAR T cell product (CTL119, containing a humanized form of FMC63). In this phase I study, 9 of 16 (56\%) patients had complete remissions

\section{Box 1 | Assays designed to monitor anti-CAR immunity}

\begin{abstract}
Cellular immunity Cytotoxicity assays

Cytotoxicity assays measure the killing of chimeric antigen receptor (CAR)-transduced target cells (including T cells and autologous lymphoid cell lines) by ex vivo expanded T cells and/or mononuclear cells from peripheral blood samples via stimulation with irradiated CAR-expressing targets. Such assays typically use ${ }^{51} \mathrm{Cr}$ labelling for quantification of target cell death after co-culture for 4-6 hours $^{10,24,27,99}$.
\end{abstract}

\section{Degranulation assays}

Degranulation assays measure CD107a (also known as Lamp1) surface expression, which indicates recent secretion of cytolytic vesicles, on ex vivo expanded patient-derived T cells from peripheral blood samples co-cultured with irradiated CAR-expressing $T$ cells using flow cytometry ${ }^{10,156}$.

\section{In vitro proliferation assays}

In vitro proliferation assays measure the ex vivo proliferation of patient-derived T cells after stimulation with irradiated CAR-expressing target cells or CAR-peptide mixtures typically through ${ }^{3} \mathrm{H}$-thymidine incorporation or flow cytometric assessment (dilution of a proliferation dye such as carboxyfluorescein diacetate succinimidyl ester or similar) ${ }^{26}$.

\section{Enzyme-Linked Immunosorbent Spot (ELISpot) assays}

ELISpot assays detect IFN $\gamma$ released by ex vivo expanded patient-derived CAR-specific T cells. These cells are typically derived from peripheral blood mononuclear cells followed by 2-week expansion in the presence of irradiated CAR-expressing T cells and IL-2. After co-culture, these cells are exposed to either irradiated CAR-expressing target cells or CAR-derived peptide pools. This approach has the advantage of enabling the identification of the distinct protein fragments recognized by anti-CAR T cells ${ }^{28,29,103}$.

\section{Intracellular cytokine staining and flow cytometry}

Intracellular cytokine staining and flow cytometry involve the detection of IFN $\gamma$ or other cytokine-producing T cells among peripheral blood mononuclear cells after stimulation with mouse-derived CAR sequence-spanning peptides (or potentially non-mouse CAR peptides). This approach uses intracellular staining with fluorochrome-labelled antibodies typically including but not limited to IFN $\gamma$, TNF, and/or IL-2 and/or extracellular staining of activation markers such as CD137 and CD40 ligand followed by flow cytometry for quantification purposes ${ }^{19,21}$.

\section{T cell receptor (TCR) repertoire analysis}

TCR repertoire analysis involves either next-generation sequencing or TCR V $\beta$ spectratyping using flow cytometry. This approach enables the identification of expanded T cell clones in vivo based on individual TCR complementarity defining regions or TCR V $\beta$ usage. Such analysis can be used to detect infused clones if TCR repertoire analysis is conducted before infusion?.

\section{Humoral anti-CAR immunity}

\section{Enzyme-linked immunosorbent assays (ELISAs)}

ELISAs are standardized assays that enable the semi-quantitative analysis of human anti-mouse antibodies (HAMAs) ${ }^{17}$. To this end, patient serum is added to mouse immunoglobulin-coated wells. After washing, human immunoglobulins bound to the mouse immunoglobulins are visualized using secondary antibody staining with an anti-human lgG typically linked to a reporter enzyme (such as horseradish peroxidase). Subsequently, enzyme-mediated luminescence or colour changes in a solution are monitored for quantification. Standards run in the same assay enable the estimation of HAMA levels.

\section{Cell-based flow cytometry}

Cell-based flow cytometry involves the incubation of CAR-expressing target cells (such as Jurkat T cells or Chinese hamster ovary (CHO) cells) with patient serum samples, followed by washing and secondary staining for anti-human $\mathrm{lgG}$. Additional steps are required to remove the HAMA signal and thus identify specific anti-CAR/idiotype antibodies unrelated to HAMA ${ }^{9,40,86,142}$.

\section{ELISA anti-drug antibody bridging assays}

ELISA anti-drug antibody bridging assays use two soluble CAR proteins, one that binds to the vessel's surface while the second is linked to a reporter such as an alkaline phosphatase or horseradish peroxidase. This complex only becomes fixed during the wash stages in the presence of an anti-CAR antibody. Additional steps are needed to remove the HAMA signal. This approach does not enable the identification of specific conformational CAR epitopes, which would require anchoring of the entire CAR to a cell membrane $e^{10,17,31,157}$. 
at 1 month. However, in the same trial, 22/22 patients with CAR T cell-naive disease were in complete remission at this time point after receiving the CTL119 CAR $\mathrm{T}$ cell product ${ }^{64}$. Whether the relatively low rate of complete remissions in patients who received reinfusions reflects an immune response to epitopes shared between mouse and humanized CAR transgenes or is due to other mechanisms of resistance remains unknown ${ }^{29,64-66,149}$.

Targeting an alternative antigen is another approach that might circumvent an anti-CAR T cell response if attributed to specific epitopes within a given $\mathrm{scFv}$ or another specific part of the CAR construct. Among these strategies, CD22-directed CAR T cells are currently the most established alternative for B cell malignancies. Thus far, CAR T cells targeting CD22 containing a fully human $\mathrm{scFv}$ have demonstrated a high degree of efficacy in patients with disease relapse on anti-CD19 CAR T cells with mouse scFvs and are equally effective regardless of CD19 expression ${ }^{150-152}$.

\section{Conclusions}

CAR T cells are currently an important therapy for patients with certain relapsed and/or refractory haematological malignancies. Despite robust reported levels of efficacy, anti-CAR immune responses are associated with treatment failure, although direct causation has, in most studies, not been established. Nevertheless, the depletion of endogenous B cells by either direct CAR targeting or lymphodepletion can reduce the risk of immune responses to CAR T cells. However, CAR $T$ cells directed to non-B cell malignancies, such as solid tumours, could induce both T cell and B cell responses, thus increasing the risk of immunogenicity (FIG. 2). This issue adds to the hurdles of designing effective CAR immunotherapies for solid tumours, where - among other challenges - a balance between the induction of potent endogenous immunity to cancer cells and responses against transgenic CAR T cells is required.
Developing technologies that improve the safety and/or functionality of CAR T cells, such as suicide genes or cytokine self-support, might also introduce new immunogenic risks. Moreover, the emergence and development of allogeneic donor-derived CAR T cells has drawn attention to the issue of immunogenicity. Indeed, the potential success of 'universal' off-the-shelf CAR T cells merits the inclusion of strategies designed to avoid immune recognition, protect against allospecific immunity and/or reduce the risk of immunogenic responses. CAR constructs with humanized scFvs and/or other less immunogenic components might also reduce the risk of anti-CAR immunity. In the clinic, intensified lymphodepletion remains vital to mitigate or modulate anti-CAR immune responses and promote better CAR T cell persistence.

Efforts to decrease the incidence and/or severity of anti-CAR immune responses should be accompanied by appropriate monitoring of immunogenicity in patients receiving CAR T cells in clinical trials. As we report, anti-CAR immunity has been investigated in fewer than a third of CAR T cell trials with available data (Supplementary Table 1). Additionally, many of the assays used to date (BOX 1) lack both standardization and validation, thus limiting the strength of correlations and performance comparisons. We strongly advocate for the inclusion of CAR-associated immunogenicity analysis in both preclinical and clinical investigations to enable both immunogenic responses and their implications to be studied in detail. The more widespread use of CAR T cells supports the need to monitor anti-CAR immunity and demands vigilance regarding possible CAR-related immunogenicity in order to ensure the safety and efficacy of CAR T cells in patients with cancer. This knowledge will increase our understanding of the clinical relevance of immunogenicity and inform the development of future CAR T cell strategies.

Published online 25 February 2021
1. June, C. H. \& Sadelain, M. Chimeric antigen receptor therapy. N. Engl. J. Med. 379, 64-73 (2018).

2. Rafiq, S., Hackett, C. S. \& Brentjens, R. J. Engineering strategies to overcome the current roadblocks in CAR T cell therapy. Nat. Rev. Clin. Oncol. 17, 147-167 (2020).

3. MacKay, M. et al. The therapeutic landscape for cells engineered with chimeric antigen receptors. Nat. Biotechnol. 38, 233-244 (2020).

4. Elsallab, M., Levine, B. L., Wayne, A. S. \& Abou-EI-Enein, M. CAR T-cell product performance in haematological malignancies before and after marketing authorisation. Lancet Oncol. 21 e104-e116 (2020).

5. Scholler, J. et al. Decade-long safety and function of retroviral-modified chimeric antigen receptor T cells. Sci. Transl. Med. 4, 132ra53 (2012).

6. Brudno, J. N. \& Kochenderfer, J. N. Recent advances in CAR T-cell toxicity: mechanisms, manifestations and management. Blood Rev. 34, 45-55 (2019).

7. Fucá, G., Reppel, L., Landoni, E., Savoldo, B. \& Dotti, G. Enhancing chimeric antigen receptor T cell efficacy in solid tumors. Clin. Cancer Res. 26 2444-2451 (2020)

8. Schmidts, A. \& Maus, M. V. Making CAR T cells a solid option for solid tumors. Front. Immunol. 9, 2593 (2018).

9. Jensen, M. C. et al. Antitransgene rejection responses contribute to attenuated persistence of adoptively transferred CD20/CD19-specific chimeric antigen receptor redirected T cells in humans. Biol. Blood Marrow Transplant. 16, 1245-1256 (2010).
10. Lamers, C. H. J. et al. Immune responses to transgene and retroviral vector in patients treated with ex vivo-engineered T cells. Blood 117, 72-82 (2011).

11. Riddell, S. R. et al. T-cell mediated rejection of gene-modified HIV-specific cytotoxic T lymphocytes in HIV-infected patients. Nat. Med. 2, 216-223 (1996).

12. Klee, G. G. Human anti-mouse antibodies. Arch. Pathol. Lab. Med. 124, 921-923 (2000).

13. Blanco, I. et al. Antiidiotypic response against murine monoclonal antibodies reactive with tumor-associated antigen TAG-72. J. Clin. Immunol. 17, 96-106 (1997).

14. Jaffers, G. J. et al. Monoclonal antibody therapy. Anti-idiotypic and non-anti-idiotypic antibodies to OKT3 arising despite intense immunosuppression. Transplantation 41, 572-578 (1986).

15. Sege, K. \& Peterson, P. A. Use of anti-idiotypic antibodies as cell-surface receptor probes. Proc. Natl Acad. Sci. USA 75, 2443-2447 (1978).

16. Kershaw, M. H. et al. A phase I study on adoptive immunotherapy using gene-modified T cells for ovarian cancer. Clin. Cancer Res. 12, 6106-6115 (2006).

17. Maus, M. V. et al. T cells expressing chimeric antigen receptors can cause anaphylaxis in humans. Cancer Immunol. Res. 1, 26-31 (2013).

18. Baudouin, V. et al. Anaphylactic shock caused by immunoglobulin E sensitization after retreatment with the chimeric anti-interleukin-2 receptor monoclonal antibody basiliximab. Transplantation 76, 459-463 (2003).
19. Awasthi, R. et al. Tisagenlecleucel cellular kinetics, dose, and immunogenicity in relation to clinical factors in relapsed/refractory DLBCL. Blood Adv. 4, 560-572 (2020).

20. European Medicines Agency. EMA - Yescarta / axicabtagene ciloleucel - Assessment report. https:// www.ema.europa.eu/en/documents/assessmentreport/yescarta-epar-public-assessment-report_en.pdf (2018).

21. Mueller, K. T. et al. Clinical pharmacology of tisagenlecleucel in B-cell acute lymphoblastic leukemia. Clin. Cancer Res. 24, 6175-6184 (2018).

22. European Medicines Agency. EMA - Kymriah / tisagenlecleucel - Assessment Report. https://www. ema.europa.eu/en/documents/assessment-report/ kymriah-epar-public-assessment-report_en.pdf (2018).

23. Kite Pharma. Kite Pharma - Tecartus / brexucabtagene autoleucel - Full prescribing information. https://www. fda.gov/vaccines-blood-biologics/cellular-gene-therapy products/tecartus-brexucabtagene-autoleucel (2020).

24. Ghorashian, S. et al. Enhanced CAR T cell expansion and prolonged persistence in pediatric patients with ALL treated with a low-affinity CD19 CAR. Nat. Med. 25, 1408-1414 (2019).

25. Till, B. G. et al. CD20-specific adoptive immunotherapy for lymphoma using a chimeric antigen receptor with both CD28 and 4-1BB domains: pilot clinical trial results. Blood 119, 3940-3950 (2012).

26. Lee, D. W. et al. T cells expressing CD19 chimeric antigen receptors for acute lymphoblastic leukaemia in children and young adults: a phase 1 doseescalation trial. Lancet 385, 517-528 (2015). 
27. Turtle, C. J. et al. CD19 CAR-T cells of defined $\mathrm{CD4}^{+}: \mathrm{CD} 8^{+}$composition in adult $\mathrm{B}$ cell $\mathrm{ALL}$ patients. J. Clin. Invest. 126, 2123-2138 (2016).

28. Turtle, C. J. et al. Immunotherapy of non-Hodgkin's lymphoma with a defined ratio of CD8+ and CD4+ CD19-specific chimeric antigen receptor-modified T cells. Sci. Transl. Med. 8, 355ra116 (2016).

29. Brudno, J. N. et al. Safety and feasibility of anti-CD19 CAR T cells with fully human binding domains in patients with B-cell lymphoma. Nat. Med. 26, 270-280 (2020)

30. Maude, S. L. et al. Tisagenlecleucel in children and young adults with B-cell lymphoblastic leukemia. N. Engl. J. Med. 378, 439-448 (2018).

31. Neelapu, S. S. et al. Axicabtagene ciloleucel CAR T-cell therapy in refractory large B-cell lymphoma. N. Engl. J. Med. 377, 2531-2544 (2017).

32. Mahadeo, K. M. et al. Management guidelines for paediatric patients receiving chimeric antigen receptor T cell therapy. Nat. Rev. Clin. Oncol. 16, 45-63 (2019).

33. Shah, N. N. \& Fry, T. J. Mechanisms of resistance to CAR T cell therapy. Nat. Rev. Clin. Oncol. 16 372-385 (2019)

34. Majzner, R. G. \& Mackall, C. L. Tumor antigen escape from CAR T-cell therapy. Cancer Discov. 8 1219-1226 (2018).

35. Gauthier, J. et al. Factors associated with outcomes after a second CD19-targeted CAR T-cell infusion for refractory B cell malignancies. Blood https://doi.org 10.1182/blood.2020006770 (2020).

36. Ali, S. A. et al. T cells expressing an anti-B-cell maturation antigen chimeric antigen receptor cause remissions of multiple myeloma. Blood 128 , 1688-1700 (2016).

37. Cohen, A. D. et al. B cell maturation antigen-specific CAR T cells are clinically active in multiple myeloma. J. Clin. Invest. 129, 2210-2221 (2019).

38. Raje, N. et al. Anti-BCMA CAR T-cell therapy bb212 in relapsed or refractory multiple myeloma. N. Engl. J. Med. 380, 1726-1737 (2019).

39. Brudno, J. N. et al. T cells genetically modified to express an anti-B-cell maturation antigen chimeric antigen receptor cause remissions of poor-prognosis relapsed multiple myeloma. J. Clin. Oncol. 36, 2267-2280 (2018).

40. Xu, J. et al. Exploratory trial of a biepitopic CAR T-targeting B cell maturation antigen in relapsed/ refractory multiple myeloma. Proc. Natl Acad. Sci. USA 116, 9543-9551 (2019).

41. Yan, Z. et al. A combination of humanised anti-CD19 and anti-BCMA CAR T cells in patients with relapsed or refractory multiple myeloma: a single-arm, phase 2 trial. Lancet Haematol. 6, e521-e529 (2019).

42. Zhao, W.-H. et al. A phase 1 , open-label study of LCAR-B38M, a chimeric antigen receptor $\mathrm{T}$ cell therapy directed against $B$ cell maturation antigen, in patients with relapsed or refractory multiple myeloma J. Hematol. Oncol. 11, 141 (2018).

43. Maciocia, P. M. et al. Targeting the T cell receptor $\beta$-chain constant region for immunotherapy of T cell malignancies. Nat. Med. 23, 1416-1423 (2017).

44. Gomes-Silva, D. et al. CD7-edited T cells expressing a CD7-specific CAR for the therapy of T-cell malignancies. Blood 130, 285-296 (2017).

45. Rasaiyaah, J., Georgiadis, C., Preece, R., Mock, U. \& Qasim, W. TCR $\alpha /$ CD3 disruption enables CD3-specific antileukemic T cell immunotherapy. JCl Insight 3, e99442 (2018).

46. Mamonkin, M., Rouce, R. H., Tashiro, H. \& Brenner, M. K. A T-cell-directed chimeric antigen receptor for the selective treatment of T-cell malignancies. Blood 126,983-992 (2015).

47. Hill, L. C. et al. Safety and anti-tumor activity of CD5 CAR T-cells in patients with relapsed/refractory T-cell malignancies. Blood 134, 199-199 (2019).

48. Hege, K. M. et al. Safety, tumor trafficking and immunogenicity of chimeric antigen receptor (CAR)-T cells specific for TAG-72 in colorectal cancer J. Immunother. Cancer 5, 22 (2017)

49. Lamers, C. H. et al. Treatment of metastatic renal cell carcinoma with CAIX CAR-engineered T cells: clinical evaluation and management of on-target toxicity. Mol. Ther. 21, 904-912 (2013).

50. Gonzales, N. Minimizing immunogenicity of the SDR-grafted humanized antibody CC49 by genetic manipulation of the framework residues. Mol. Immunol. 40, 337-349 (2003).

51. Maher, J., Brentjens, R. J., Gunset, G., Rivière, I. \& Sadelain, M. Human T-lymphocyte cytotoxicity and proliferation directed by a single chimeric TCRzeta / CD28 receptor. Nat. Biotechnol. 20, 70-75 (2002).
52. Imai, C. et al. Chimeric receptors with 4-1BB signaling capacity provoke potent cytotoxicity against acute lymphoblastic leukemia. Leukemia 18, 676-684 (2004).

53. Savoldo, B. et al. CD28 costimulation improves expansion and persistence of chimeric antigen receptor-modified T cells in lymphoma patients. J. Clin. Invest. 121, 1822 (2011).

54. Kalos, M. et al. T cells with chimeric antigen receptors have potent antitumor effects and can establish memory in patients with advanced leukemia. Sci. Transl. Med. 3, 95ra73 (2011).

55. Grupp, S. A. et al. Chimeric antigen receptor-modified T cells for acute lymphoid leukemia. N. Engl. J. Med. 368, 1509-1518 (2013).

56. Ahmed, N. et al. Human epidermal growth factor receptor 2 (HER2) -specific chimeric antigen receptor-modified T cells for the immunotherapy of HER2-positive sarcoma. J. Clin. Oncol. 33, 1688-1696 (2015).

57. Ahmed, N et al. HER2-specific chimeric antigen receptor-modified virus-specific T cells for progressive glioblastoma. JAMA Oncol. 3, 1094-1101 (2017).

58. Moritz, D., Wels, W., Mattern, J. \& Groner, B. Cytotoxic T lymphocytes with a grafted recognition specificity for ERBB2-expressing tumor cells. Proc. Natl Acad. Sci. USA 91, 4318-4322 (1994).

59. Wels, W., Harwerth, I. M., Mueller, M., Groner, B. \& Hynes, N. E. Selective inhibition of tumor cell growth by a recombinant single-chain antibody-toxin specific for the erbB-2 receptor. Cancer Res. 52, 6310-6317 (1992).

60. Hegde, M. et al. Tumor response and endogenous immune reactivity after administration of HER2 CAR $T$ cells in a child with metastatic rhabdomyosarcoma. Nat. Commun. 11, 3549 (2020).

61. Gargett, T. et al. GD2-specific CAR T cells undergo potent activation and deletion following antigen encounter but can be protected from activation induced cell death by PD-1 blockade. Mol. Ther. 24 1135-1149 (2016).

62. Haas, A. R. et al. Phase I study of lentiviral-transduced chimeric antigen receptor-modified T cells recognizing mesothelin in advanced solid cancers. Mol. Ther. 27, 1919-1929 (2019).

63. Junghans, R. P. et al. Phase I trial of anti-PSMA designer CAR-T cells in prostate cancer: possible role for interacting interleukin 2-T cell pharmacodynamics as a determinant of clinical response. Prostate 76, 1257-1270 (2016).

64. Maude, S. L. et al. Durable remissions with humanized CD19-targeted chimeric antigen receptor (CAR) modified T cells in CAR-naive and CAR-exposed children and young adults with relapsed/refractory acute lymphoblastic leukemia. Blood 130 1319-1319 (2017)

65. Cao, J et al. Potent anti-leukemia activities of humanized CD 19-targeted chimeric antigen receptor $\mathrm{T}$ (CAR-T) cells in patients with relapsed/refractory acute lymphoblastic leukemia. Am. J. Hematol. 93 , 851-858 (2018)

66. Zhao, Y. et al. Treatment with humanized selective CD19CAR-T cells shows efficacy in highly treated B-ALL patients who have relapsed after receiving murine-based CD 19CAR-T therapies. Clin. Cancer Res. 25, 5595-5607 (2019)

67. Holliger, P. \& Hudson, P. J. Engineered antibody fragments and the rise of single domains. Nat. Biotechnol. 23, 1126-1136 (2005).

68. Clarke, S. C. et al. Multispecific antibody development platform based on human heavy chain antibodies Front. Immunol. 9, 3037 (2019).

69. Li, N., Fu, H., Hewitt, S. M., Dimitrov, D. S. \& Ho, M. Therapeutically targeting glypican-2 via single-domain antibody-based chimeric antigen receptors and immunotoxins in neuroblastoma. Proc. Natl Acad. Sci. USA 114, E6623-E6631 (2017).

70. Schneider D et al. A unique human immunoglobulin heavy chain variable domain-only CD33 CAR for the treatment of acute myeloid leukemia. Front. Oncol. 8 539 (2018).

71. Mikkilineni, L. et al. T cells expressing an antiB-cell maturation antigen (BCMA) chimeric antigen receptor with a fully-human heavy-chain-only antigen recognition domain induce remissions in patients with relapsed multiple myeloma. Blood 134, 3230-3230 (2019).

72. Lam, N. et al. Anti-BCMA chimeric antigen receptors with fully human heavy-chain-only antigen recognition domains. Nat. Commun. 11, 283 (2020).

73. Zhang, T., Barber, A. \& Sentman, C. L. Generation of antitumor responses by genetic modification of primary human T cells with a chimeric NKG2D receptor. Cancer Res. 66, 5927-5933 (2006)

74. Frazao, A. et al. NKG2D/NKG2-ligand pathway offers new opportunities in cancer treatment. Front. Immunol. 10, 661 (2019)

75. Deng, X. et al. Antitumor activity of NKG2D CAR-T cells against human colorectal cancer cells in vitro and in vivo. Am. J. Cancer Res. 9, 945-958 (2019).

76. Kahlon, K. S. et al. Specific recognition and killing of glioblastoma multiforme by interleukin 13-Zetakine redirected cytolytic T cells. Cancer Res. 64 9160-9166 (2004)

77. Sengupta, S., Thaci, B., Crawford, A. C. \& Sampath, P. Interleukin-13 receptor alpha 2-targeted glioblastom immunotherapy. BioMed. Res. Int. 2014, 952128 (2014).

78. Brown, C. E. et al. Regression of glioblastoma after chimeric antigen receptor T-cell therapy. N. Engl. J. Med. 375, 2561-2569 (2016).

79. Pameijer, C. R. J. et al. Conversion of a tumor-binding peptide identified by phage display to a functional chimeric T cell antigen receptor. Cancer Gene Ther 14, 91-97 (2007).

80. Whilding, L. M. et al. Targeting of aberrant $\alpha \vee \beta 6$ integrin expression in solid tumors using chimeric antigen receptor-engineered T cells. Mol. Ther. $\mathbf{2 5}$, 259-273 (2017).

81. Muniappan, A., Banapour, B., Lebkowski, J. \& Talib, S. Ligand-mediated cytolysis of tumor cells: use of heregulin- $\zeta$ chimeras to redirect cytotoxic T lymphocytes. Cancer Gene Ther. 7, 128-134 (2000).

82. Cho, J. H., Collins, J. J. \& Wong, W. W. Universal chimeric antigen receptors for multiplexed and logical control of T cell responses. Cell 173, 1426-1438.e1 (2018).

83. Lohmueller, J. J., Ham, J. D., Kvorjak, M. \& Finn, O. J. mSA2 affinity-enhanced biotin-binding CAR T cells for universal tumor targeting. Oncoimmunology 7 , e1368604 (2017)

84. Hudecek, M. et al. Receptor affinity and extracellular domain modifications affect tumor recognition by ROR 1-specific chimeric antigen receptor T cells. Clin. Cancer Res. 19, 3153-3164 (2013).

85. Guest, R. D. et al. The role of extracellular spacer regions in the optimal design of chimeric immune receptors. J. Immunother. 28, 203-211 (2005).

86. Till, B. G. et al. Adoptive immunotherapy for indolent non-Hodgkin lymphoma and mantle cell lymphoma using genetically modified autologous CD20-specific T cells. Blood 112, 2261-2271 (2008)

87. Jonnalagadda, M. et al. Chimeric antigen receptors with mutated IgG4 Fc spacer avoid Fc receptor binding and improve $T$ cell persistence and antitumor efficacy. Mol. Ther. 23, 757-768 (2015)

88. Hombach, A., Hombach, A. A. \& Abken, H. Adoptive immunotherapy with genetically engineered $T$ cells: modification of the IgG1 Fc 'spacer' domain in the extracellular moiety of chimeric antigen receptors avoids 'off-target' activation and unintended initiation of an innate immune response. Gene Ther. 17 1206-1213 (2010).

89. Shum, T., Kruse, R. L. \& Rooney, C. M. Strategies for enhancing adoptive T-cell immunotherapy against solid tumors using engineered cytokine signaling and other modalities. Expert Opin. Biol. Ther. 18, 653-664 (2018).

90. Conlon, K. C. et al. Redistribution, hyperproliferation, activation of natural killer cells and CD8 T cells, and cytokine production during first-in-human clinical tria of recombinant human interleukin-15 in patients with cancer. J. Clin. Oncol. 33, 74-82 (2015).

91. Chen, Y. et al. Eradication of neuroblastoma by T cells redirected with an optimized GD2-specific chimeric antigen receptor and interleukin-15. Clin. Cancer Res. 25, 2915-2924 (2019).

92. Hu, B. et al. Augmentation of antitumor immunity by human and mouse CAR T cells secreting IL-18. Cell Rep. 20, 3025-3033 (2017).

93. Ma, X. et al. Interleukin-23 engineering improves CAR T cell function in solid tumors. Nat. Biotechnol. 38, 448-459 (2020).

94. Kim, T.-S. \& Shin, E-C. The activation of bystander $\mathrm{CD} 8+\mathrm{T}$ cells and their roles in viral infection. Exp. Mol. Med. 51, 1-9 (2019).

95. Pacheco, Y. et al. Bystander activation and autoimmunity. J. Autoimmun. 103, 102301 (2019).

96. Heczey, A. et al. Anti-GD2 CAR-NKT cells in patients with relapsed or refractory neuroblastoma: an interim analysis. Nat. Med. 26, 1686-1690 (2020)

97. Shum, T. et al. Constitutive signaling from an engineered IL7 receptor promotes durable tumor 
elimination by tumor-redirected T cells. Cancer Discov. 7. 1238-1247 (2017).

98. Kagoya, Y. et al. A novel chimeric antigen receptor containing a JAK-STAT signaling domain mediates superior antitumor effects. Nat. Med. 24, 352-359 (2018).

99. Berger, C., Flowers, M. E., Warren, E. H. \& Riddell, S. R. Analysis of transgene-specific immune responses that limit the in vivo persistence of adoptively transferred HSV-TK-modified donor T cells after allogeneic hematopoietic cell transplantation. Blood 107, 2294-2302 (2006).

100. Di Stasi, A. et al. Inducible apoptosis as a safety switch for adoptive cell therapy. N. Engl. J. Med. 365, 1673-1683 (2011)

101. Zhou, X. et al. Inducible caspase-9 suicide gene controls adverse effects from alloreplete T cells after haploidentical stem cell transplantation. Blood $\mathbf{1 2 5}$ 4103-4113 (2015)

102. Wang, X. et al. A transgene-encoded cell surface polypeptide for selection, in vivo tracking, and ablation of engineered cells. Blood 118, 1255-1263 (2011).

103. Arber, C. et al. The immunogenicity of virus-derived $2 \mathrm{~A}$ sequences in immunocompetent individuals. Gene Ther. 20, 958-962 (2013).

104. Mizuguchi, H., Xu, Z., Ishii-Watabe, A., Uchida, E. \& Hayakawa, T. IRES-dependent second gene expression is significantly lower than cap-dependent first gene expression in a bicistronic vector. Mol. Ther. 1 , 376-382 (2000)

105. Wiebking, V. et al. Metabolic engineering generates a transgene-free safety switch for cell therapy. Nat. Biotechnol. 38, 1441-1450 (2020).

106. Ren, J. et al. Multiplex genome editing to generate universal CAR T cells resistant to PD1 inhibition. Clin. Cancer Res. 23, 2255-2266 (2017).

107. Lu, Y. et al. Safety and feasibility of CRISPR-edited $\mathrm{T}$ cells in patients with refractory non-small-cell lung cancer. Nat. Med. 26, 732-740 (2020).

108. Stadtmauer, E. A. et al. CRISPR-engineered T cells in patients with refractory cancer. Science 367 eaba7365 (2020).

109. Qasim, W. et al. Molecular remission of infant B-ALL after infusion of universal TALEN gene-edited CAR T cells. Sci. Transl. Med. 9, eaaj2013 (2017).

110. Torikai, H. et al. Toward eliminating HLA class I expression to generate universal cells from allogeneic donors. Blood 122, 1341-1349 (2013).

111. Kagoya, Y. et al. Genetic ablation of HLA class I, class II, and the T cell receptor enables allogeneic $\mathrm{T}$ cells to be used for adoptive T cell therapy. Cancer Immunol. Res. 8, 926-936 (2020).

112. Charlesworth, C. T. et al. Identification of preexisting adaptive immunity to Cas9 proteins in humans. Nat. Med. 25, 249-254 (2019).

113. Simhadri, V. L. et al. Prevalence of pre-existing antibodies to CRISPR-associated nuclease Cas9 in the USA population. Mol. Ther. Methods Clin. Dev. 10 105-112 (2018)

114. Fu, Y. et al. High-frequency off-target mutagenesis induced by CRISPR-Cas nucleases in human cells. Nat. Biotechnol. 31, 822-826 (2013).

115. Zhang, X.-H., Tee, L. Y., Wang, X.-G., Huang, Q.-S. \& Yang, S.-H. Off-target effects in CRISPR/Cas9mediated genome engineering. Mol. Ther. Nucleic Acids 4, e264 (2015).

116. Abou-El-Enein, M. et al. Human genome editing in the clinic: new challenges in regulatory benefit-risk assessment. Cell Stem Cell 21, 427-430 (2017).

117. Wagner, D. L. et al. High prevalence of Streptococcus pyogenes Cas9-reactive T cells within the adult human population. Nat. Med. 25, 242-248 (2019).

118. Li, A. et al. AAV-CRISPR gene editing is negated by pre-existing immunity to Cas9. Mol. Ther. $\mathbf{2 8}$, 1432-1441 (2020)

119. Depil, S., Duchateau, P., Grupp, S. A., Mufti, G. $\&$ Poirot, L. 'Off-the-shelf' allogeneic CAR T cells: development and challenges. Nat. Rev. Drug Discov. 19, 185-199 (2020)

120. Suchin, E. J. et al. Quantifying the frequency of alloreactive $T$ cells in vivo: new answers to an old question. J. Immunol. 166, 973-981 (2001)

121. DeWolf, S. et al. Quantifying size and diversity of the human T cell alloresponse. JCI Insight 3, e 121256 (2018).

122. Spierings, E. et al. Multicenter analyses demonstrate significant clinical effects of minor histocompatibility antigens on GvHD and GvL after HLA-matched related and unrelated hematopoietic stem cell transplantation. Biol. Blood Marrow Transplant. 19 1244-1253 (2013).
123. Benjamin, R. et al. Genome-edited, donor-derived allogeneic anti-CD 19 chimeric antigen receptor $T$ cells in paediatric and adult B-cell acute lymphoblastic leukaemia: results of two phase 1 studies. Lancet 396 1885-1894 (2020).

124. Gornalusse, G. G. et al. HLA-E-expressing pluripotent stem cells escape allogeneic responses and lysis by NK cells. Nat. Biotechnol. 35, 765-772 (2017).

125. Xu, H. et al. Targeted disruption of HLA genes via CRISPR-Cas9 generates iPSCs with enhanced immune compatibility. Cell Stem Cell 24, 566-578.e7 (2019).

126. Lanza, R., Russell, D. W. \& Nagy, A. Engineering universal cells that evade immune detection. Nat. Rev. Immunol. 19, 723-733 (2019).

127. Deuse, T. et al. Hypoimmunogenic derivatives of induced pluripotent stem cells evade immune rejection in fully immunocompetent allogeneic recipients. Nat. Biotechnol. 37, 252-258 (2019).

128. Mo, F. et al. Engineered off-the-shelf therapeutic T cells resist host immune rejection. Nat. Biotechnol. 39, 56-63 (2021)

129. Muranski, P. et al. Increased intensity lymphodepletion and adoptive immunotherapy - how far can we go? Nat. Clin. Pract. Oncol. 3, 668-681 (2006).

130. Miller, J. S. et al. Successful adoptive transfer and in vivo expansion of human haploidentical NK cells in patients with cancer. Blood 105 , 3051-3057 (2005).

131. Tzannou, I. et al. Off-the-shelf virus-specific T cells to treat BK virus, human herpesvirus 6 , cytomegalovirus, Epstein-Barr virus, and adenovirus infections after allogeneic hematopoietic stem-cell transplantation. J. Clin. Oncol. 35, 3547-3557 (2017).

132. Leen, A. M. et al. Multicenter study of banked third-party virus-specific T cells to treat severe viral infections after hematopoietic stem cell transplantation. Blood 121, 5113-5123 (2013).

133. Melenhorst, J. J. et al. Allogeneic virus-specific T cells with HLA alloreactivity do not produce GVHD in human subjects. Blood 116, 4700-4702 (2010).

134. Pule, M. A. et al. Virus-specific T cells engineered to coexpress tumor-specific receptors: persistence and antitumor activity in individuals with neuroblastoma. Nat. Med. 14, 1264-1270 (2008)

135. Liu, E. et al. Use of CAR-transduced natural killer cells in CD19-positive lymphoid tumors. N. Engl. J. Med. 382, 545-553 (2020).

136. Heczey, A. et al. Invariant NKT cells with chimeric antigen receptor provide a novel platform for safe and effective cancer immunotherapy. Blood 124, 2824-2833 (2014).

137. Deniger, D. C. et al. Bispecific T-cells expressing polyclonal repertoire of endogenous $\gamma \delta$ T-cell receptors and introduced $C D 19$-specific chimeric antigen receptor. Mol. Ther. 21, 638-647 (2013)

138. Yakoub-Agha, I. et al. Management of adults and children undergoing chimeric antigen receptor T-cell therapy: best practice recommendations of the European Society for Blood and Marrow Transplantation (EBMT) and the Joint Accreditation Committee of ISCT and EBMT (JACIE). Haematologica 105, 297-316 (2020).

139. Kansagra, A. J. et al. Clinical utilization of chimeric antigen receptor T-cells (CAR-T) in B-cell acute lymphoblastic leukemia (ALL) - an expert opinion from the European Society for Blood and Marrow Transplantation (EBMT) and the American Society for Blood and Marrow Transplantation (ASBMT). Bone Marrow Transpl. 54, 1868-1880 (2019).

140. Jain, T. et al. Use of chimeric antigen receptor T cell therapy in clinical practice for relapsed/refractory aggressive B cell non-Hodgkin lymphoma: an exper panel opinion from the American Society for Transplantation and Cellular Therapy. Biol. Blood Marrow Transpl. 25, 2305-2321 (2019).

141. Ramos, C. A. et al. Clinical and immunological responses after $\mathrm{CD} 30$-specific chimeric antigen receptor-redirected lymphocytes. J. Clin. Invest. 127, 3462-3471 (2017)

142. Potthoff, B., McBlane, F., Spindeldreher, S. \& Sickert, D. A cell-based immunogenicity assay to detect antibodies against chimeric antigen receptor expressed by tisagenlecleucel. J. Immunol. Methods 476, 112692 (2020)

143. Jahn, E.-M. \& Schneider, C. K. How to systematically evaluate immunogenicity of therapeutic proteins regulatory considerations. N. Biotechnol. 25 280-286 (2009)

144. European Medicines Agency. Immunogenicity assessment of biotechnology-derived therapeutic proteins (EMEA/CHMP/BMWP/14327/2006). https://www.ema.europa.eu/en/immunogenicityassessment-biotechnology-derived-therapeuticproteins (2018).

145. Shankar, G. et al. Assessment and reporting of the clinical immunogenicity of therapeutic proteins and peptides-harmonized terminology and tactical recommendations. AAPS J. 16, 658-673 (2014).

146. US Food and Drug Administration. Immunogenicity Testing of Therapeutic Protein Products - Developing and Validating Assays for Anti-Drug Antibody Detection (FDA-2009-D-0539; 03/15/2019). https://www.fda.gov/regulatory-information/ search-fda-guidance-documents/immunogenicity testing-therapeutic-protein-products-developingand-validating-assays-anti-drug (2020).

147. Zhang, $\mathrm{H}$. et al. Lymphopenia and interleukin-2 therapy alter homeostasis of $\mathrm{CD} 4{ }^{+} \mathrm{CD} 25^{+}$regulatory T cells. Nat. Med. 11, 1238-1243 (2005).

148. Maude, S. L. et al. Efficacy and safety of humanized chimeric antigen receptor (CAR)-modified T cells targeting CD19 in children with relapsed/refractory ALL. Blood 126, 683 (2015).

149. Li, M.-Y. et al. Secondary donor-derived humanized CD19-modified CAR-T cells induce remission in relapsed/refractory mixed phenotype acute leukemia after allogeneic hematopoietic stem cell transplantation: a case report. Biomark. Res. 8, 36 (2020).

150. Shah, N. N. et al. CD4/CD8 T-cell selection affects chimeric antigen receptor (CAR) T-cell potency and toxicity: updated results from a phase I anti-CD22 CAR T-cell trial. J. Clin. Oncol. 38, 1938-1950 (2020).

151. Pan, J. et al. CD22 CAR T-cell therapy in refractory or relapsed B acute lymphoblastic leukemia. Leukemia 33, 2854-2866 (2019).

152. Fry, T. J. et al. CD22-targeted CAR T cells induce remission in B-ALL that is naive or resistant to CD19-targeted CAR immunotherapy. Nat. Med. 24 , 20-28 (2018).

153. Yang, Y. \& Wilson, J. M. Clearance of adenovirusinfected hepatocytes by MHC class I-restricted CD4 ${ }^{+}$CTLs in vivo. J. Immunol. 155, 2564-2570 (1995).

154. Restifo, N. P., Dudley, M. E. \& Rosenberg, S. A. Adoptive immunotherapy for cancer: harnessing the T cell response. Nat. Rev. Immunol. 12, 269-281 (2012).

155. Scott, A. M., Allison, J. P. \& Wolchok, J. D. Monoclonal antibodies in cancer therapy. Cancer Immunol. Res. 12 (2012).

156. Kochenderfer, J. N. et al. B-cell depletion and remissions of malignancy along with cytokineassociated toxicity in a clinical trial of anti-CD 19 chimeric-antigen-receptor-transduced T cells. Blood 119, 2709-2720 (2012).

157. Beatty, G. L. et al. Mesothelin-specific chimeric antigen receptor mRNA-engineered T cells induce anti-tumor activity in solid malignancies. Cancer Immunol. Res. 2 112-120 (2014).

\section{Acknowledgements}

The work of N.A. and M.H. is supported by the US NIH PHS grant U54-CA23256 and by Stand Up to Cancer-St Baldrick's Paediatric Dream Team Translational Research Grant (SU2C-AACR-DT1113). The work of A.S.W. is supported by the US National Cancer Institute [P30CA014089]. The content of this publication does not necessarily reflect the views of policies of the Department of Health and Human Services nor does its mention of trade names, commercial products or organizations imply endorsement by the U.S. Government. The authors thank Catherine Gillespie (Baylor College of Medicine, USA) for her assistance in editing the manuscript and thank Naomi Taylor and Christopher Chien (Pediatric Oncology Branch, National Cancer Institute, NIH, USA) for their critical review of the figures.

\section{Author contributions}

D.L.W., E.F. and M.A. researched data for the manuscript. All authors made a contribution to discussions of content. D.L.W., E.F., M.A.P., N.A., M.H.a., M.H.e., B.S., N.N.S., C.J.T., A.S.W. and M.A. wrote the manuscript. All authors edited and/or reviewed the manuscript prior to submission.

\section{Competing interests}

M.A.P. has acted as an advisor for Mesoblast and Novartis, has received research funding from Adaptive and Miltenyi, and has received honoraria for educational activities from Miltenyi and Novartis. N.A. has acted as a consultant for Adaptimmune and continues to consult for Equillium (pro 
bono) and The Children's Cancer Hospital Egypt 57357 on medical education and research development, has received one-time royalties from Celgene and Cell Medica, and is a named inventor on patents and patent applications in the field of chimeric antigen receptor (CAR) T cell therapy owned by Baylor College of Medicine. M. Hamieh is listed on patents relating to CAR technologies and has received royalties from Atara Biotherapeutics, Fate Therapeutics and Takeda Pharmaceuticals. M. Hegde is a named inventor on patents and patent applications in the field of CAR T cell therapy owned by Baylor College of Medicine. M.R. has acted as consultant of $\mathrm{AbClon}$, Bristol-Myers Squibb (BMS) Mesoblast, NanoString, and Novartis, has received research support from Adaptive and Miltenyi, and is listed on patents relating to CAR technologies and receives royalties from Novartis and Tmunity. B.S. has acted as a consultant of Tessa Therapeutics, has received research funding from Bellicum Pharmaceuticals, Bluebird Bio, Cell Medica and Tessa Therapeutics, and is listed on patents in the field of CAR and
T cell receptor (TCR) therapy. C.J.T. has served on the scientific advisory boards of ArsenalBio, Caribou Biosciences, Century Therapeutics, Eureka Therapeutics, Myeloid Therapeutics, Precision Biosciences and T-CURX, has acted as an ad hoc consultant of Allogene, Amgen, AstraZeneca, Nektar Therapeutics and PACT Pharma, has stock/options in ArsenalBio, Caribou Biosciences, Eureka Therapeutics, Myeloid Therapeutics and Precision Biosciences, has received research funding from AstraZeneca, Juno Therapeutics/BMS Minerva, Nektar Therapeutics and TCR2 Therapeutics, and is listed on a patent licensed to Juno Therapeutics. A.S.W. has received research support from Kite Pharma and Institut de Recherches Internationales Servier. E.F., D.L.W., N.N.S. and M.A. declare no competing interests.

\section{Peer review information}

Nature Reviews Clinical Oncology thanks Marcela Maus and the other, anonymous, reviewer(s) for their contribution to the peer review of this work.
Publisher's note

Springer Nature remains neutral with regard to jurisdictional claims in published maps and institutional affiliations.

\section{Review criteria}

We conducted a literature screen and identified 120 clinical trial publications describing the use of chimeric antigen receptor (CAR) T cells, out of which 34 reported on the investigation of immunogenicity using laboratory assays (Box 1). A complete list of these trials, including information on the assays used to characterize immunogenicity, CAR design, trial outcomes and preconditioning regimens, is available in Supplementary Table 1. Data cut-off July 15, 2020.

Supplementary information

The online version contains supplementary material available at https://doi.org/10.1038/s41571-021-00476-2. 